\title{
Organization of Corticostriatal and Corticoamygdalar Projections Arising from the Anterior Inferotemporal Area TE of the Macaque Monkey: A Phaseolus vulgaris Leucoagglutinin Study
}

\author{
K. Cheng, K. S. Saleem, and K. Tanaka \\ The Institute of Physical and Chemical Research (RIKEN), Wako, Saitama 351-01, Japan
}

Corticostriatal and corticoamygdalar projections arising from area TE of the macaque monkey were studied by focal injections of the anterograde tracer Phaseolus vulgaris leucoagglutinin into the dorsolateral and ventromedial subdivisions of the anterior TE (TEad and TEav, respectively). This approach yielded several new results. First, the global distributions of labeled terminals revealed that both TEad and TEav projected to the ventrocaudal striatum, including the tail of the caudate nucleus and the adjacent ventral putamen, and the dorsolateral aspect of the deep amygdaloid nuclei. TEav also projected to the medial basal nucleus of the amygdala and the ventral striatum. Second, the reconstructed single axons $(n=18)$ demonstrated that some axons originating from TEav or TEad projected simultaneously to the ventrocaudal striatum and the dorsolateral aspect of the deep amygdaloid nuclei by giving off collaterals. TEav axons projected to the medial basal nucleus of the amygdala also had collaterals projecting to the perirhinal cortex or area TG. And third, it was revealed that the axons originating from a focal TEav or TEad projected to a restricted territory (3.4-3.6 $\mathrm{mm}$ rostrocaudally) in the ventrocaudal striatum with four to six dispersed, rostrocaudally elongated, rodlike modules. Individual axons with multiple arbors innervated many of these modules. These findings add the evidence that the anterior part of TE is anatomically heterogeneous and suggest that the deep amygdaloid nuclei may be functionally dissociated, with the dorsolateral aspect more closely related to the ventrocaudal striatum and the medial basal nucleus more closely related to the perirhinal cortex.

Key words: macaque monkey; inferotemporal cortex; area TE; column; striatum; amygdala; perirhinal cortex; corticostriatal projection; corticoamygdalar projection; PHA-L; single axon; axon collaterals; arbor
Area TE of the inferotemporal cortex of the macaque monkey is crucial for recognition and discrimination of visual images of objects (for review, see Gross, 1973; Dean, 1976). Neurons in TE selectively respond to complex visual features, and TE neurons with similar stimulus selectivity cluster in columnar regions (for review, see Tanaka, 1993, 1996). TE projects to several polysensory areas, including the perirhinal cortex, striatum, and amygdala. The projections from the anterior part of TE to the striatum and amygdala were analyzed in the present study.

The striatum receives projections from visual and sensorimotor areas and sends outputs to the pallidum and substantia nigra, which in turn project to the thalamus and then to motor areas of the frontal cortex. This cortico-basal ganglia-thalamo-cortical loop is thought to integrate visual or sensorimotor information with behavioral significance and to influence motor output (for review, see Parent and Hazrati, 1995). The amygdala receives highly processed sensory information from all sensory modalities and in turn projects to the hypothalamus, thalamus, striatum, and hippocampal formation (for review, see Amaral et al., 1992) and is thought to be crucial in certain forms of associative and emotional memories (for review, see Gaffan, 1992; LeDoux, 1992; Gallagher and Chiba, 1996).

Received June 19, 1997; revised Aug. 5, 1997; accepted Aug. 7, 1997.

This work was supported by the Frontier Research Program, The Institute of Physical and Chemical Research (RIKEN), Japan. We thank W. Suzuki and A. H. Asiya Begum for surgical and histological assistance and two anonymous reviewers for helpful comments.

Correspondence should be addressed to Dr. K. Cheng, Laboratory for Cognitive Brain Mapping, Brain Science Institute, The Institute of Physical and Chemical Research (RIKEN), Hirosawa 2-1, Wako, Saitama 351-01, Japan.

Copyright (C) 1997 Society for Neuroscience $0270-6474 / 97 / 177902-24 \$ 05.00 / 0$
Previous studies have described the projections from TE to the striatum and amygdala (Whitlock and Nauta, 1956; Jones and Powell, 1970; Kemp and Powell, 1970; Herzog and Van Hoesen, 1976; Yeterian and Van Hoesen, 1978; Aggleton et al., 1980; Turner et al., 1980; Van Hoesen et al., 1981; Iwai and Yukie, 1987; Iwai et al., 1987; Saint-Cyr et al., 1990; Webster et al., 1991, 1993; Baizer et al., 1993), but these descriptions were limited to the global features of the projections. In the present study, we used focal injections of Phaseolus vulgaris leucoagglutinin (PHA-L) to elucidate the following three aspects of the projections. First, because it has been suggested that the anterior part of $\mathrm{TE}$ is a heterogeneous area that is composed of dorsolateral (TEad) and ventromedial (TEav) divisions based on anatomical (Yukie et al., 1992; Saleem et al., 1995; Saleem and Tanaka, 1996) and inactivation (for review, see Horel, 1996) studies, we compared the projections from anatomically defined TEad and TEav to the striatum and amygdala. Second, because the columnar organization has been shown in TE (for review, see Tanaka, 1993, 1996), we analyzed projection patterns from a small area in TEad and TEav by localizing the injection site to a size comparable to that of TE columns (Fujita et al., 1992). Finally, and most importantly, focal injections of PHA-L made it possible to trace single corticostriatal and corticoamygdalar axons. Information about branching and arborizing patterns of single axons, which has been lacking because of technical limitation (cf. DiFiglia et al., 1978), is of special value for understanding better the functional organization of corticostriatal and corticoamygdalar systems. The results from reconstructed single axons were emphasized in the present study. 
Some of the present results have appeared elsewhere in abstract form (Cheng et al., 1993).

\section{MATERIALS AND METHODS}

Four Japanese monkeys (Macaca fuscata), weighing between 3.3 and 4.6 $\mathrm{kg}$, were used. A single PHA-L injection was made in TEad of the right hemisphere of two monkeys and in TEav of the right hemisphere of the other two monkeys. Three of these four PHA-L cases were also used in another study conducted in this laboratory (Saleem and Tanaka, 1996).

Surgery and injection. The methods for surgery and injection have been described in detail by Saleem et al. (1993) and Saleem and Tanaka (1996). Briefly, PHA-L was delivered during aseptic surgery under general anesthesia. After an initial introduction of atropine sulfate (0.1 $\mathrm{mg} / \mathrm{kg}$, i.m.), the monkey was anesthetized with ketamine hydrochloride $(12 \mathrm{mg} / \mathrm{kg}$, i.m.), followed by intraperitoneal injection of sodium pentobarbital (Nembutal, $35 \mathrm{mg} / \mathrm{kg}$ ). Tranexamic acid $(25 \mathrm{mg} / \mathrm{kg}$, i.m.) was administered to minimize bleeding. Supplemental doses of sodium pentobarbital $(9 \mathrm{mg} / \mathrm{kg}$, i.p.) were injected when necessary to maintain the surgical level of anesthesia.

After a large craniotomy was made over the temporal area, the dura was cut to expose a large extent of the superior temporal sulcus and the anterior middle temporal sulcus for determining the site of PHA-L injection. To reduce the brain volume and to facilitate access to the cortex medial to the anterior middle temporal sulcus, $20 \mathrm{ml}$ of mannitol $(20 \%)$ was intravenously injected in the two TEav cases. PHA-L $(2.5 \%$; Vector Laboratories, Burlingame, CA) was injected iontophoretically (Midgard precision current source; Stoelting), according to the procedure recommended by Gerfen and Sawchenko (1984) with some modifications (Saleem et al. 1993). After the injection of PHA-L was completed, the dura was sutured, and the wound was closed. The antibiotic piperacillin sodium $(55 \mathrm{mg} / \mathrm{kg}$, i.m.) and the analgesic ketoprofen (5 $\mathrm{mg} / \mathrm{kg}$, i.m.) were injected daily for $4-5 \mathrm{~d}$ after the surgery.

Perfusion and histology. After 16-18 d of postinjection survival, the monkey was lethally anesthetized with sodium pentobarbital and perfused through the heart with 11 of $0.9 \%$ warm heparinized saline and then $3-41$ of $4 \%$ paraformaldehyde in $0.1 \mathrm{M}$ phosphate buffer, $\mathrm{pH}$ $7.2-7.4,1-21$ of $10 \%$ sucrose in $0.1 \mathrm{M}$ phosphate buffer, and finally 11 of $20 \%$ sucrose in $0.1 \mathrm{M}$ phosphate buffer. The brain was removed immediately after the perfusion, photographed, blocked, and then put in $30 \%$ buffered sucrose at $4^{\circ} \mathrm{C}$ until it sank. Frozen tissue blocks were sectioned at 30 (case 1, TEad), 35 (case 2, TEad), and 40 (cases 3 and 4, TEav) $\mu \mathrm{m}$ thickness in the coronal plane. All sections were processed for PHA-L, and some of the PHA-L sections were counterstained for Nissl after the PHA-L analysis was completed, to facilitate demarcation of the borders of the cortical areas and layers and the amygdaloid nuclei. Transported PHA-L was visualized by an avidin-biotin immunoperoxidase method described by Saleem et al. (1993).

Data analysis. All sections were first globally analyzed for the extent of labeled terminals in the striatum, the amygdala, and the claustrum. The outlines of these structures and labeled terminals for selected sections were drawn with the aid of a camera-lucida microscope attachment $(20 \times$ or $40 \times$ magnification for structure outlines, and $100 \times$ magnification for labeled terminals). The interval between adjacent selected sections varied between cases and structures, ranging from 300 to $700 \mu \mathrm{m}$. The PHA-L-treated sections exhibited substantial shrinkage of at least $25 \%$ in the mediolateral and dorsoventral dimensions on a coronal section (Rockland et al., 1994). In the present study, the rostrocaudal extent was calculated based on the thickness of frozen sections. The shrinkage in the two dimensions on coronal sections was not corrected.

The global observation of the labeling also aided in the reconstruction of single axons by facilitating the localization of patches or clusters of terminals and axonal trunks well filled with PHA-L in the gray matter. A single axon was followed, using the camera-lucida microscope attachment, through serial sections at $200 \times$ magnification, usually starting from a segment of an axonal trunk in the vicinity of a terminal cluster in the striatum or the amygdala. From this segment, the axonal trunk was traced to two other extremes of the axon. These were distal portions of the axon with terminal specializations, that is, the varicose or stalked profile of terminal collaterals and the proximal portion of the axon entering the white matter, which in most cases could be traced back to the injection site. For all reconstructed axons, a variable number of branches were encountered during the course of the reconstruction. Each branch was then traced independently. When an axon was being traced, proximal segments of other axons in the vicinity were simultaneously traced to provide reliable landmarks for matching the axon between adjacent sections. If such nearby axons were not available, other landmarks such as blood vessels were used for the purpose of alignment. Terminal arbors were traced with morphological details such as varicosities and terminal swellings, presumably synaptic boutons, at a higher magnification $(400 \times)$. The majority of terminals in an arbor were traced to their ends, but some terminals became too faint to be followed, and in some rare cases, blind ending processes were encountered. The exact extent of an arbor, thus, may have been slightly underestimated, but no axonal branches appear to have been missed unless otherwise noted. Although all arbors (if possible) of an axon were traced with varicosities and terminal swellings, we did not focus our analysis in the present study on these morphological details.

Nomenclature of amygdaloid nuclei and cortical regions. Most of the subcortical structures including the amygdaloid nuclei were readily distinguishable in PHA-L sections under Nomarsky optics. Subdivisions of the amygdaloid nuclei described by Amaral et al. (1992) were adopted with some modifications. The basal nucleus was divided into the lateral basal nucleus (corresponding to the magnocellular and intermediate divisions of the basal nucleus of Amaral et al., 1992) and the medial basal nucleus (corresponding to the parvicellular division of the basal nucleus) on the grounds that the projections from TEav and TEad to these two nuclei appeared to be organized very differently (see Results). The periamygdaloid cortex, the central nucleus, and the accessory basal nucleus were not further subdivided, because there were no or only faintly labeled terminals in these subnuclei. The paralaminar nucleus, a very thin nucleus immediately ventral to the medial basal nucleus (Amaral et al., 1992), was identifiable but is not illustrated in the figures in the present study. The cytoarchitectonic borders between TEad and TEav and between TEav and the perirhinal cortex are based on the results of Saleem and Tanaka (1996).

\section{RESULTS}

After a brief description of PHA-L injection sites and general labeling features of axonal fibers and terminals, the results will be presented in three sections. The global distributions of terminal fields, with emphases on similarities and differences between projections from TEav and TEad to the striatum and the amygdala, will be described in the first section. Detailed patterns of projections from TEav and TEad to the striatum and the amygdala, based on serially reconstructed single axons, will then be presented in the second section. Finally, in the third section, the organization of corticostriatal projections from a focal injection in TEav or TEad will be investigated by comparing terminal arborizations of single axons with the global distributions of labeled terminals.

\section{Injection sites and general features of PHA-L labeling}

PHA-L injections in the two TEad cases were made in the cortex between the ventral lip of the superior temporal sulcus and the lateral lip of the anterior middle temporal sulcus and in the two TEav cases in the cortex just medial to the medial lip of the anterior middle temporal sulcus (Fig. $1 A-C$ ). The injection sites measured from 0.5 to $1.0 \mathrm{~mm}$ in diameter in the plane parallel to the pial surface and involved all cortical layers in three cases (cases 1, 3, and 4 ; see Fig. $1 B, C$ ) and the lower part of layer III through layer VI in the fourth case (case 2). All injection sites were well localized within TEad or TEav (Saleem and Tanaka, 1996).

Axonal branches and terminals in the gray matter in all cases were thoroughly filled with PHA-L, allowing us to visualize morphological details such as varicosities and terminal swellings (see Fig. 8B). Axonal trunks in the white matter were also satisfactorily labeled, and in most cases they could be traced back to the injection site. In addition, axonal fibers that projected to different subcortical structures or cortical areas were usually grouped together in different bundles and could be easily differentiated in serial coronal sections (Fig. 1D). 
A
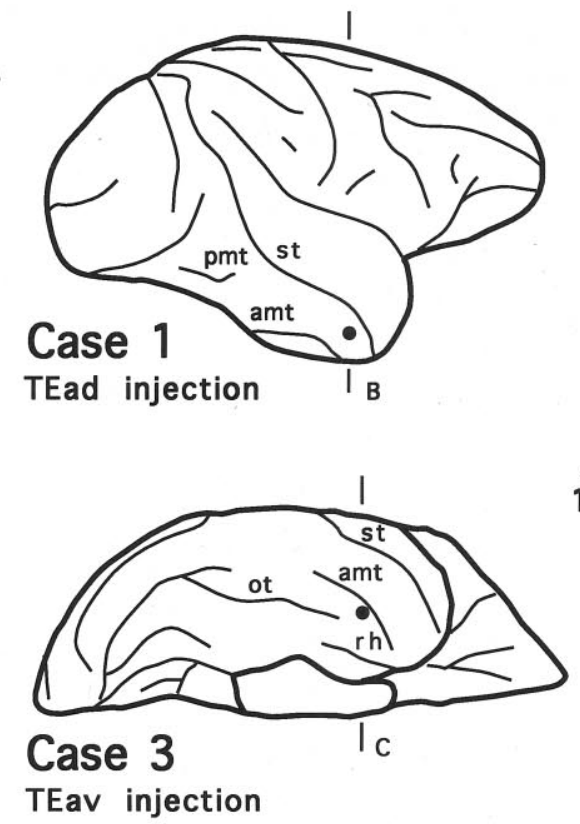

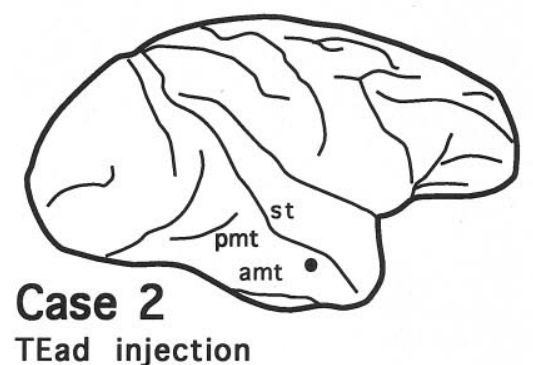

$0 \mathrm{~mm}$

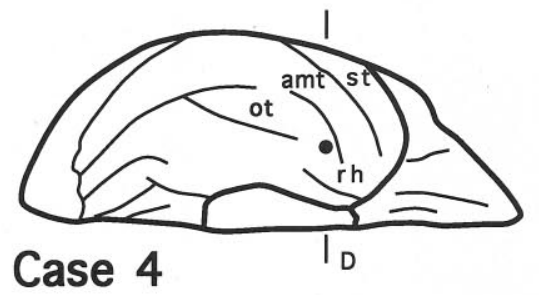

TEav injection
Figure 1. PHA-L injection sites and labeling of axonal fibers in the white matter. $A$, Schematic brain diagrams illustrating PHA-L injection sites (filled circles) in the two TEad cases (Case 1 and Case 2, lateral view) and the two TEav cases (Case 3 and Case 4, bottom view). $B, C$, Photomicrographs showing PHA-L injection sites in coronal sections in a TEad case $(B$, case 1$)$ and a TEav case $(C$, case 3$)$. The two sections were counterstained for Nissl to identify the cortical layers. $D$, Camera-lucida drawing of a coronal section from case 4. PHA-L-labeled axonal fibers in the white matter circumscribed by the shaded rectangle are shown at higher magnification by a photomicrograph (bottom panel). The two arrows indicate an axonal trunk that courses dorsally and terminates in the ventral putamen. The complete reconstruction of this axon (axon 4-1) is shown in Figure 15. The bundles of fibers on the right run dorsally and rostrally toward more rostrally located subcortical structures or cortical areas. amt, Anterior middle temporal sulcus; $o t$, occipitotemporal sulcus; pmt, posterior middle temporal sulcus; $s t$, superior temporal sulcus; $r h$, rhinal sulcus; Amy, amygdala; $C d$, caudate nucleus; $C l$, claustrum; $H$, hippocampus; $L V$, lateral ventricle; Put, putamen. The same abbreviations for sulci are used in all figures unless otherwise stated. Scale bars: $B, C, 1 \mathrm{~mm} ; D, 0.2 \mathrm{~mm}$.
B

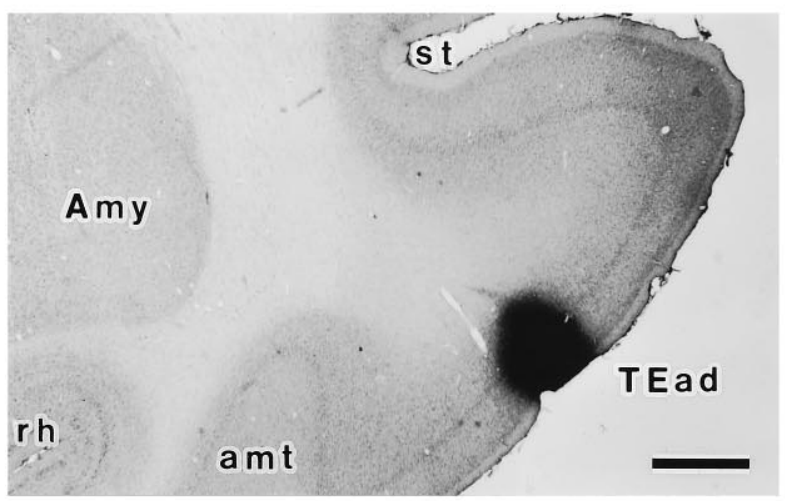

\section{C}

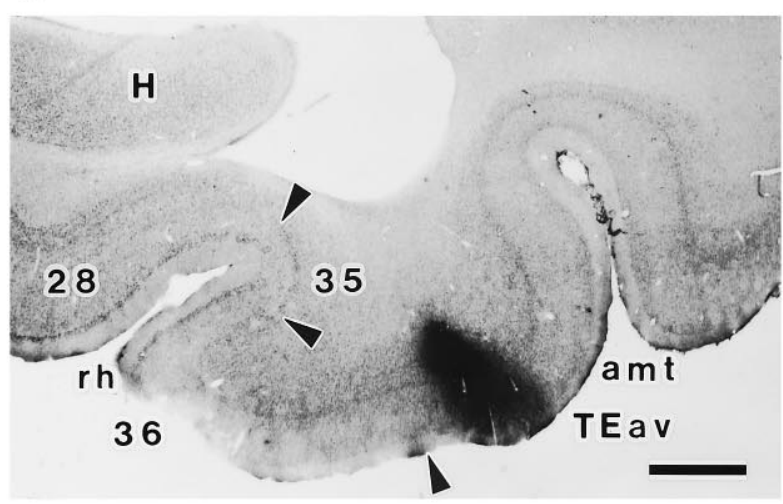

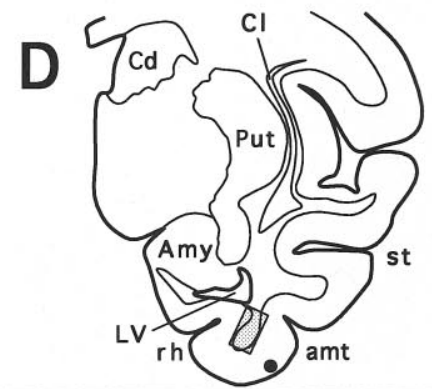

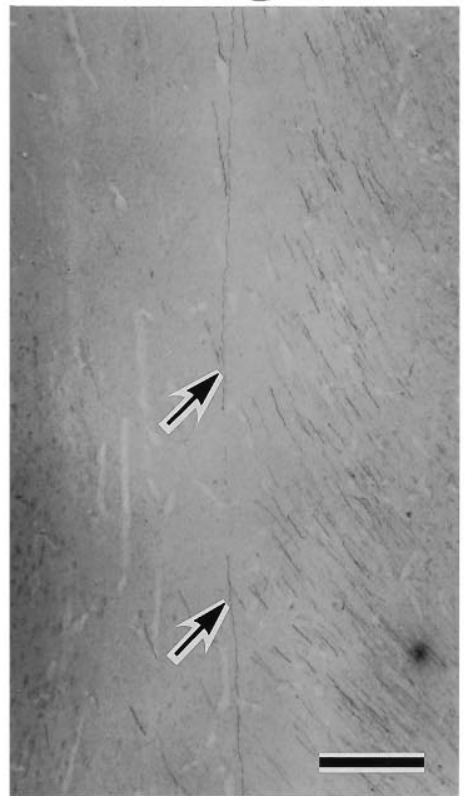

\section{Global distributions of labeled terminals: TEav versus TEad}

Projections to the striatum

As shown in Figure 2 for case 3, labeled terminals formed two separate dense zones in the striatum after TEav injections: one in the lateral aspect of the ventrocaudal striatum composed of the tail of the caudate nucleus and the adjacent ventral putamen (Fig. $2 A-D$ ), and the other in the ventral striatum composed of the nucleus accumbens, the olfactory tubercle, and the adjacent ventral part of the head of the caudate nucleus and the ventral 


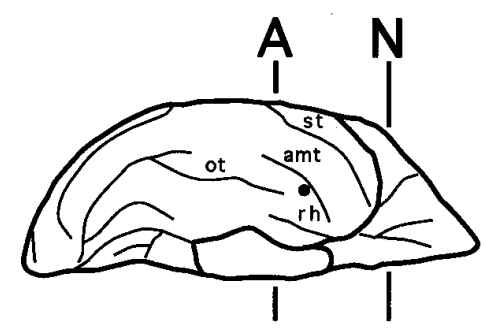

\section{Case 3 TEav injection}
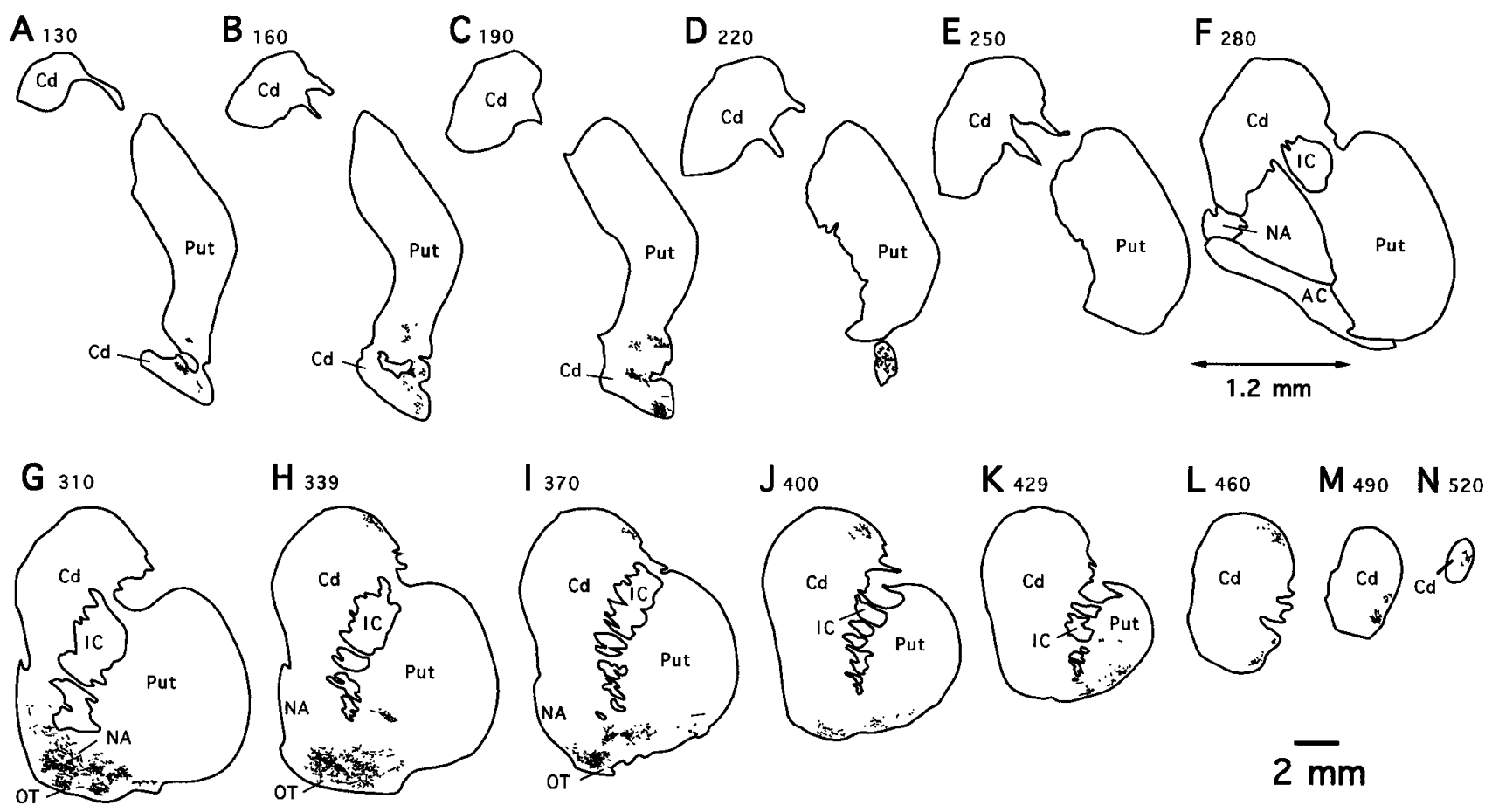

Figure 2. Camera-lucida drawings of 13 coronal section outlines showing the distribution of PHA-L-labeled terminals in the ventrocaudal striatum (composed of the tail of the caudate nucleus and adjacent ventral putamen), the ventral striatum (composed of the nucleus accumbens, olfactory tubercle, and adjacent ventral part of the head of the caudate nucleus and ventral putamen), and the remaining parts of the striatum after TEav injection (case 3). Numbers indicate serial numbers of individual sections (40 $\mu \mathrm{m}$ thick); bottom section numbers indicate posterior sections. Section outlines are illustrated caudorostrally $(A-N)$ at equal intervals $(1.2 \mathrm{~mm})$. Note the absence of labeled terminals at the level of the anterior commissure $(E, F)$. $A C$, Anterior commissure; $C d$, caudate nucleus; $I C$, internal capsule; $N A$, nucleus accumbens; $O T$, olfactory tubercle; Put, putamen.

putamen (Fig. 2G-I). The two terminal zones were separated from each other at the level of the anterior commissure (Fig. $2 E, F)$. In addition, some sparsely distributed terminals were seen more rostrally in the dorsolateral part of the head of the caudate nucleus and the lateral part of the ventral putamen (Fig. $2 \mathrm{H}-\mathrm{N}$ ). The distributions of labeled terminals in the other TEav case (case 4; data not shown) were very similar to those observed in case 3 .

As shown for case 3 in Figure $2 A-D$, the labeled terminals in the ventrocaudal striatum were distributed within a restricted total territory, which measured $\sim 2.1 \mathrm{~mm}(2.2 \mathrm{~mm}$ in case 4$)$ mediolaterally (ML), $4.2 \mathrm{~mm}(3.9 \mathrm{~mm})$ dorsoventrally (DV), and $3.5 \mathrm{~mm}(3.4 \mathrm{~mm})$ rostrocaudally $(\mathrm{RC})$. The rostrocaudal extent of terminal fields in the ventral striatum was limited to $\sim 3.0 \mathrm{~mm}$ in both cases, but the labeled terminals covered almost the full mediolateral and dorsoventral extents of the ventral striatum. In both the ventrocaudal striatum and ventral striatum, the labeled terminals were not evenly distributed within the total territory, but they formed several distinctive patches in any given section.

Injections into TEad resulted in similar distinctive patchy ter- minal fields in the ventrocaudal striatum, whereas there were no labeled terminals in the ventral striatum or other portions of the striatum. The distribution of labeled terminals for case 1 is illustrated in Figure 3, but a very similar projection pattern was observed in case 2 (see Fig. 11). Compared with the terminal fields of TEav projections, the terminal fields in the TEad cases were slightly more sparse but were distributed within a restricted territory of comparable size $(1.8 \mathrm{~mm} \mathrm{ML} \times 4.7 \mathrm{~mm} \mathrm{DV} \times 3.6 \mathrm{~mm}$ $\mathrm{RC}$ in case 1 and $2.6 \mathrm{~mm} \times 3.5 \mathrm{~mm} \times 3.5 \mathrm{~mm}$ in case 2$)$. It should be noted that the terminal fields in the ventrocaudal striatum in all four cases were very similar with respect to their RC extents (3.4-3.6 mm).

The measurements of the terminal fields in the ML and DV directions after TEav or TEad injections were comparable to those described for the corticostriatal projections based on large injections in $\mathrm{TE}$, but the RC extents were much shorter than those in the previous studies [cf. $13 \mathrm{~mm}$ (Saint-Cyr et al., 1990, measured from their Fig. 11A), $16 \mathrm{~mm}$ (Baizer et al., 1993, their Fig. 6B), and $9 \mathrm{~mm}$ (Webster et al., 1993, their Fig. 7)]. The difference in the RC extent between our cases and these previous 


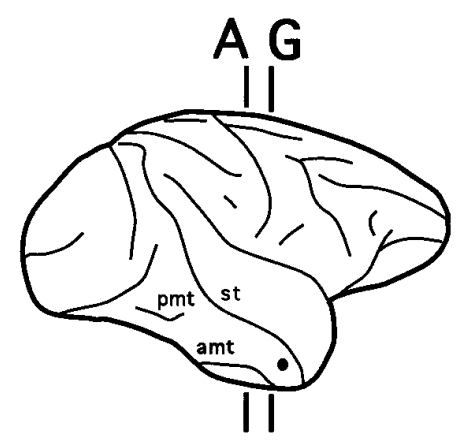

\section{Case 1 \\ TEad injection}
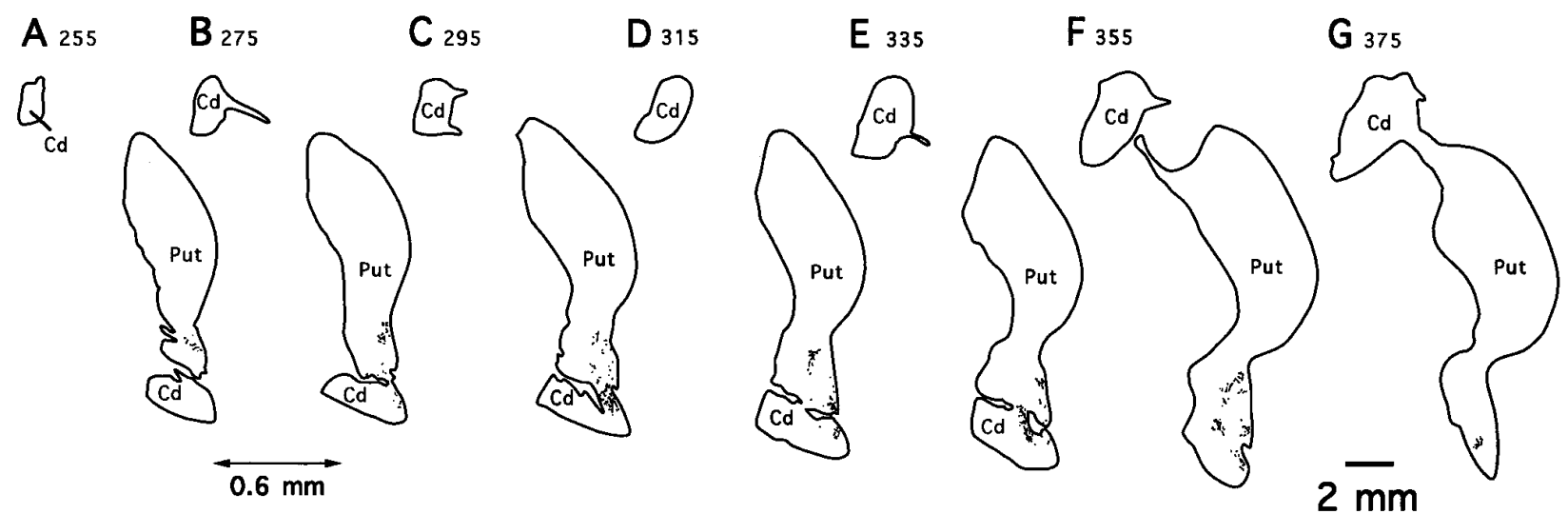

Figure 3. Camera-lucida drawings of 7 coronal section outlines showing the distribution of PHA-L-labeled terminals in the ventrocaudal striatum after TEad injection (case 1). Numbers indicate serial numbers of individual sections (30 $\mu$ m thick); bottom section numbers indicate posterior sections. Section outlines are illustrated caudorostrally $(A-G)$ at equal intervals $(0.6 \mathrm{~mm})$. Abbreviations are the same as in Figure 2.

studies may be attributable to the size and location of injection sites; their injections were much larger than ours and involved both the anterior and posterior portions of the dorsal TE.

\section{Projections to the amygdala}

The terminal fields in the amygdala formed two zones in both TEav cases, as illustrated in Figure 4 for case 3. In one zone, labeled terminals were mostly distributed in the lateral basal nucleus and the dorsomedial aspect of the lateral nucleus. Halfway into the amygdaloid complex, some sparsely but definitely labeled terminals extended into the dorsolateral aspect of the accessory basal nucleus (Fig. $4 F$ ). The labeled terminals in the accessory basal nucleus were denser and extended more medially in case 4 (see Figs. 10,12) than in case 3. This projection zone is referred to as the dorsolateral aspect of the deep amygdaloid nuclei in the present study.

The terminal fields in the dorsolateral aspect of the deep amygdaloid nuclei after TEav injections are not composed of distinctive patches, although the labeled terminals are not evenly distributed over the terminal fields (Fig. 4). A prominent characteristic of the TEav projections to the dorsolateral aspect of the deep amygdaloid nuclei was their large coverage of the rostrocaudal extent of the deep amygdaloid nuclei. The terminal field covered the rostral $80 \%$ in case 4 and the rostral $70 \%$ in case 3 of the total extent of the amygdala (4.2 of 5.4 and 3.6 of $5.2 \mathrm{~mm}$, respectively). In addition, in both TEav cases, the terminal fields in the dorsolateral aspect of the deep amygdaloid nuclei shifted ventrally toward more rostral levels (Fig. 4).

The second zone of the terminal fields of TEav projections was located in the rostral half of the medial basal nucleus in both TEav cases (see Figs. 4, 10, 12). Compared with those in the dorsolateral aspect of the deep amygdaloid nuclei, the terminal fields in the medial basal nucleus were more restricted in their rostrocaudal and mediolateral extents. Both rostrocaudally and mediolaterally, they covered about half of the total extent of the nucleus (2.0 mm ML and $2.5 \mathrm{~mm} \mathrm{RC}$ in both cases). The terminal fields covered the entire dorsoventral extent of the medial basal nucleus from its ventral extreme to its border with the lateral basal nucleus.

It should be pointed out that labeled terminals in the medial basal nucleus remained separated from those in the dorsolateral aspect of the deep amygdaloid nuclei throughout their entire rostrocaudal extents. We shall describe this separation more thoroughly in the next section, because the reconstructed single axons have demonstrated that the projection originating from TEav to the medial basal nucleus is different from that to the dorsolateral aspect of the deep amygdaloid nuclei.

In both TEav cases, no labeled terminals were observed in the central nucleus or any of the superficial amygdaloid nuclei.

After injections into TEad, labeled terminals were found only in the dorsolateral aspect of the deep amygdaloid nuclei. No labeled terminals were observed in the medial basal nucleus. Compared with the terminal fields of the projections in the two TEav cases, those from TEad appeared to be much more sparse and were distributed more laterally, mostly in the lateral nucleus, and to a lesser extent in the lateral basal nucleus (case 2, Fig. 5). There was no labeling in the accessory basal nucleus. The rostrocaudal extents of the terminal fields were also limited to about $25 \%$ (case 1 ) and $55 \%$ (case 2) of the total extent of the deep amygdaloid nuclei (1.2 of 5.0 and 2.8 of $5.3 \mathrm{~mm}$, respectively). 


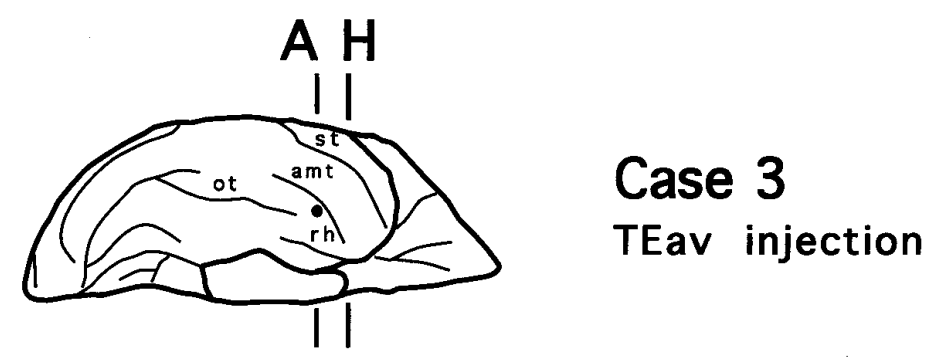

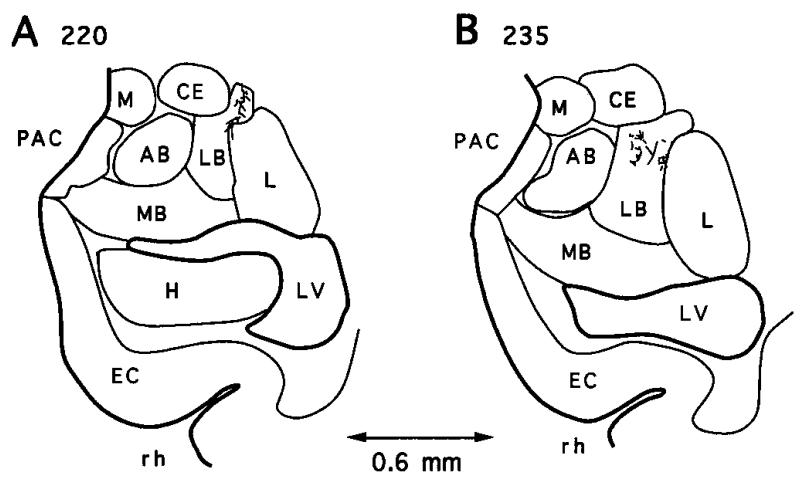

E 280

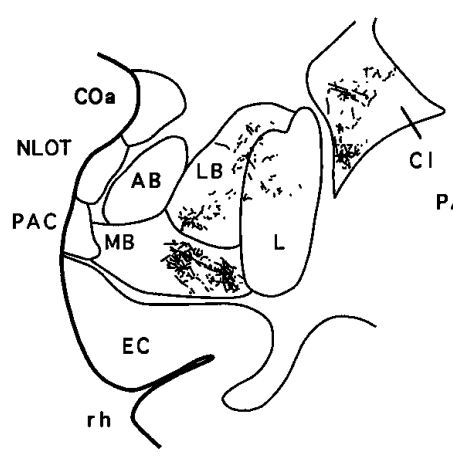

F 295

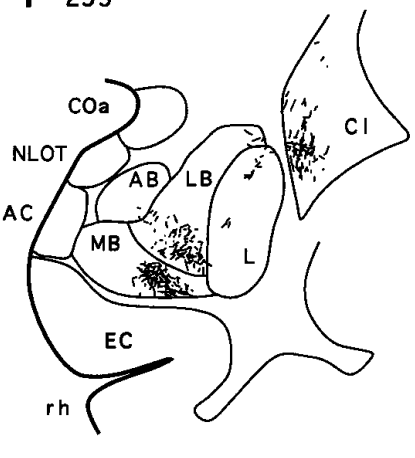

C 250

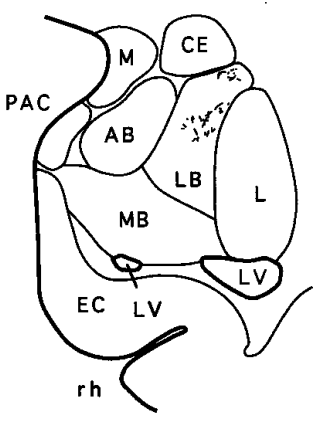

G 310

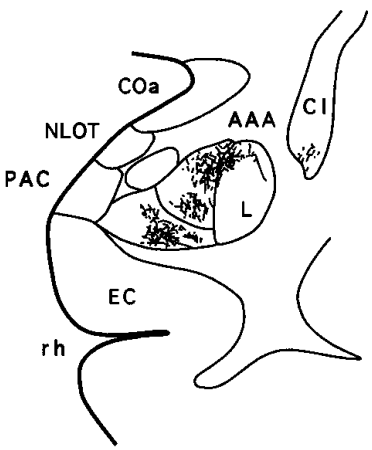

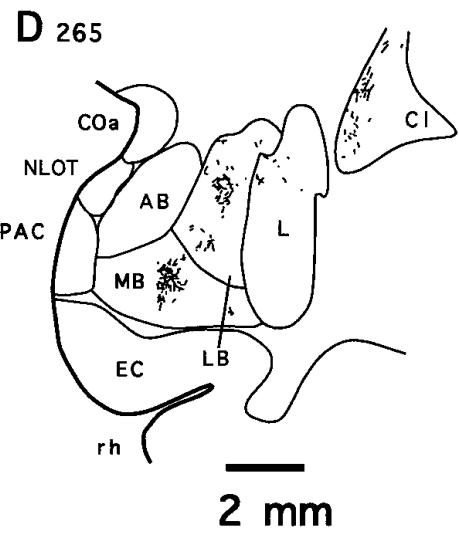

H 325

Figure 4. Camera-lucida drawings of 8 coronal section outlines showing the distribution of PHA-L-labeled terminals in the amygdala after TEav injection (case 3). Numbers indicate serial numbers of individual sections (40 $\mu \mathrm{m}$ thick); bottom section numbers indicate posterior sections. Section outlines are illustrated caudorostrally $(A-H)$ at equal intervals $(0.6 \mathrm{~mm})$. $A A A$, Anterior amygdaloid area; $A B$, accessory basal nucleus; $C E$, central nucleus; $C O a$, anterior cortical nucleus; $E n$, endopiriform nucleus; $L$, lateral nucleus; $L B$, lateral basal nucleus; $M$, medial nucleus; $M B$, medial basal nucleus; $N L O T$, nucleus of the lateral olfactory tract; $P A C$, periamygdaloid cortex; $P$ ir, piriform cortex; $D A N$, deep amygdaloid nuclei; $C d$, caudate nucleus; $C l$, claustrum; $E C$, entorhinal cortex; $H$, hippocampus; $L V$, lateral ventricle; $P u t$, putamen; $r h$, rhinal sulcus.

\section{Projections to the claustrum}

In close proximity to the terminal fields in the amygdala, the projections from both TEav and TEad to the claustrum were observed. Labeled terminals were densely distributed in the ventromedial extreme of the claustrum in all four cases. There were no apparent differences in the density and distribution of the terminal fields between TEav and TEad cases (Figs. 4, 5). The rostrocaudal extents of labeled terminals in the claustrum measured $\sim 2.0 \mathrm{~mm}$ in all cases, and in each case, the terminal field in the claustrum appeared at about the same rostrocaudal level as that where labeled terminals in the dorsolateral aspect of the amygdala were distributed (Figs. $4 D-G, 5 B-E$ ).

The observation that TEav and TEad projected equally well to the claustrum provided further evidence that differences in the strength and distribution of projections from TEav and TEad to the striatum and amygdala were not attributable to the difference in the size of the injection or the labeling quality between TEav and TEad cases. The similarity or difference in the internal representation between TEav and TEad remains to be elucidated, but the sizes of TEav and TEad are not very different [the ratio of the areal extent of TEav to that of TEad was estimated as $\sim 55: 45$; also see the unfolded maps of TEav and TEad injections by Saleem and Tanaka (1996, their Figs. 7, 8)]. Thus, it is our contention that the apparent difference in the amount of coverage of the rostrocaudal extent of the dorsolateral aspect of the deep amygdaloid nuclei between TEav and TEad injections are genuine properties of corticoamygdalar projections arising from different subdivisions within the anterior part of TE.

\section{Projection patterns of the reconstructed single axons}

The projection patterns of individual axons described in the present study are based on 18 axons with collaterals and arboriza- 


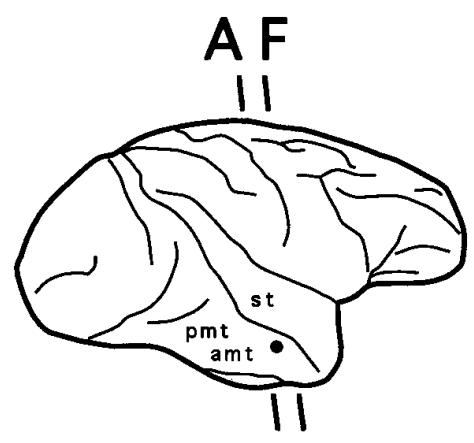

\section{Case 2 \\ TEad injection}
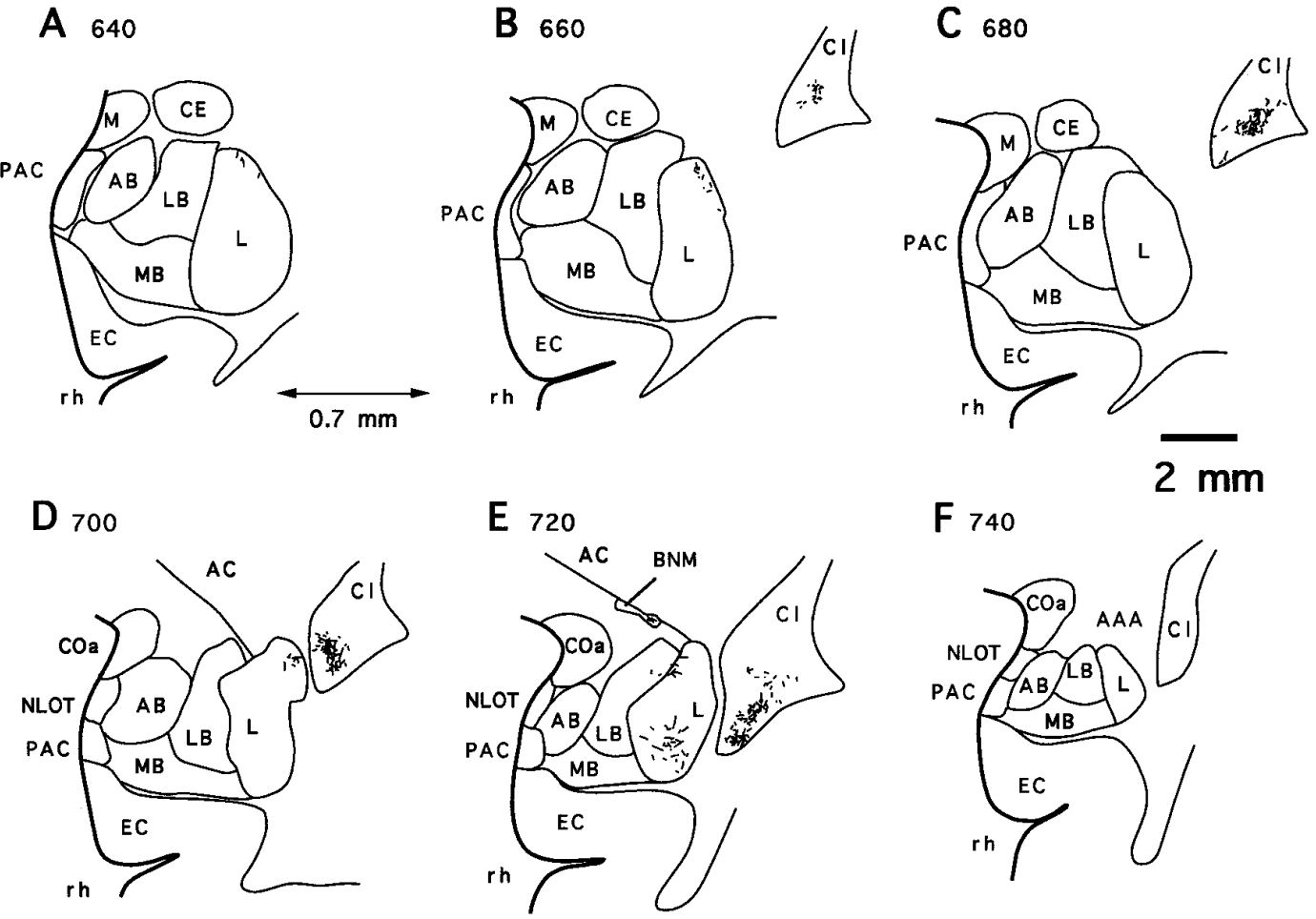

Figure 5. Camera-lucida drawings of 6 coronal section outlines showing the distribution of PHA-L-labeled terminals in the amygdala after TEad injection (case 2). Numbers indicate serial numbers of individual sections (35 $\mu \mathrm{m}$ thick); bottom section numbers indicate posterior sections. Section outlines are illustrated caudorostrally $(A-F)$ at equal intervals $(0.7 \mathrm{~mm})$. Note the absence of labeled terminals in the medial basal nucleus $(M B)$. $A C$, Anterior commissure; $B N M$, basal nucleus of Meynert. Other abbreviations are the same as in Figure 4.

tions within the striatum and/or the amygdala that were nearly completely reconstructed (referred to as completely reconstructed axons). Thirteen of the 18 axons were reconstructed from the TEav cases, and the remaining five were reconstructed from the TEad cases. We reconstructed more single axons from the TEav cases because the branching patterns of TEav axons displayed more variety, and TEad axons showed only a fraction of the variety of TEav axons. In addition, six TEav axons were partially reconstructed to verify the branching patterns of certain axons (referred to as partially reconstructed axons). Figure 6 summarizes the projections of single axons from TEav and TEad to the striatum and amygdala based on branching patterns of axon collaterals, including (1) TEav and TEad axons projecting to both the ventrocaudal striatum and the dorsolateral aspect of the deep amygdaloid nuclei, (2) TEav axons projecting to both the medial basal nucleus of the amygdala and cortex (perirhinal cortex or area TG), and (3) TEav or TEad axons projecting only to the dorsolateral aspect of the deep amygdaloid nuclei, the ventrocaudal striatum, or the ventral striatum. A few axons had collaterals projecting further to other structures but were not investigated in the present study. The detailed projection patterns of these individually reconstructed axons are described below.

\section{Dual projection of TEav and TEad axons to the ventrocaudal striatum and amygdala}

Figure 7 shows an axon reconstructed from a TEav case (axon 4-2). After leaving the injection site, the axon enters the white matter, where it follows a course lateral to the lateral ventricle and heads toward the ventrocaudal striatum. On entering the ventral putamen, the main axonal trunk bifurcates (Fig. 7, arrow at section 129) and then gives off four collaterals (Fig. 7, arrows at sections 107 and 113). Two collaterals divide further to form multiple terminal arbors in the tail of the caudate nucleus and the ventral putamen (Fig. 7, arl-ar9). Some of these arbors are richly ramified and result in highly complex plexuses (Fig. 7, ar1, ar6, ar7), whereas the others are poorly ramified. The axonal fibers in the ventrocaudal striatum have a smooth appearance, whereas the labeled terminals display varied degrees of varicosities and ter- 
A

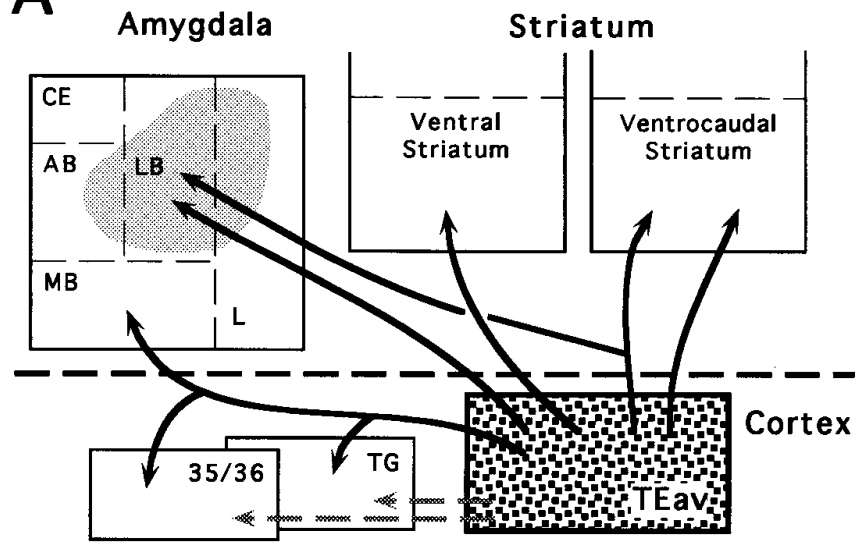

B

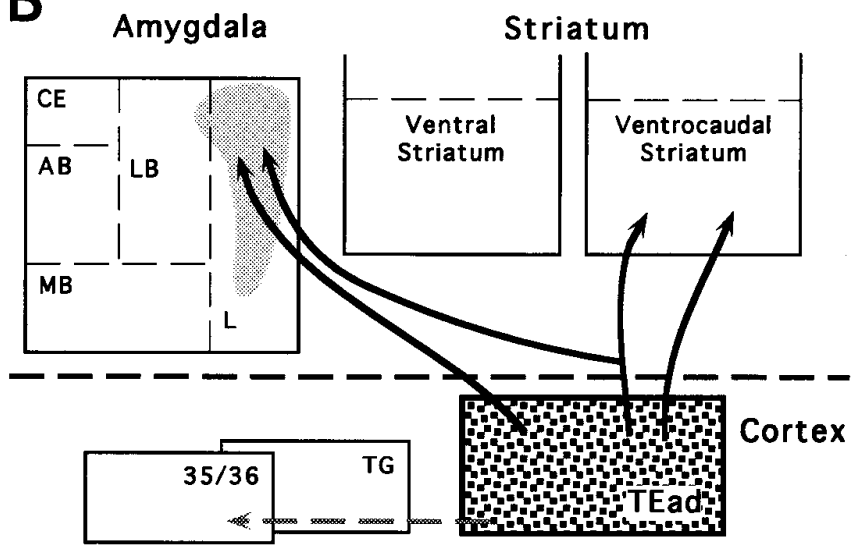

Figure 6. Schematic diagrams showing the projections of single axons from TEav $(A)$ and TEad $(B)$ to the striatum and amygdala. The shaded areas in the amygdala indicate the relative locations of the projections to the lateral nucleus, lateral basal nucleus, and accessory basal nucleus, which are regarded as single projection zones (dorsolateral aspect of the deep amygdaloid nuclei) and are distinguished from the medial basal nucleus (see Results). Arrows with bifurcating branches indicate axons with collaterals that terminate in two or more subcortical structures or cortical areas. Other arrows show axons that arborize within a single subcortical structure or cortical area. Shaded arrows show the projections from TEav and TEad to the perirhinal cortex (areas 35 and 36) and area TG, which have been described in detail in another paper (Saleem and Tanaka, 1996). Those axon collaterals that project further to other structures are not shown (see Results). $A B$, Accessory basal nucleus; $C E$, central nucleus; $L$, lateral nucleus; $L B$, lateral basal nucleus; $M B$, medial basal nucleus; $35 / 36$, areas 35 and 36 of the perirhinal cortex; $T G$, area TG.

minal swellings, presumably the synaptic boutons (Figs. $8 B, D, F$, $9 A-E)$. These morphological details, however, were not described further here. The third collateral turns medially and courses rostrally and ventrally toward the medial aspect of the ventrocaudal striatum. After traveling briefly in the white matter between the ventral putamen and the dorsolateral aspect of the amygdala, it enters the dorsal part of the lateral nucleus of the amygdala. As the collateral runs through the lateral nucleus caudorostrally, it remains devoid of terminations for a few hundred micrometers. Then, unlike axon collaterals in the ventrocaudal striatum, it emits boutons en passant along its course and finally develops into a highly beaded terminal plexus within the lateral nucleus (Fig. 7, ar10; also see Figs. $8 G, H, 9 F)$. The fourth collateral courses dorsoposteriorly and medially, traversing the ventral putamen and both the external and internal segments of the pallidum. We were unable to reconstruct this collateral fully, because it runs beyond the most posterior section cut in this case. Presumably, it projects to the thalamic nuclei or even below the thalamic level.

Another TEav axon (axon 4-3) that projects to both the ventrocaudal striatum and the dorsolateral aspect of the deep amygdaloid nuclei is shown in Figure 10. Like axon 4-2, axon 4-3 also bifurcates after entering the tail of the caudate nucleus (Fig. 10, arrow at section 74). However, unlike axon 4-2, the two branches of axon 4-3 only innervate the tail of the caudate nucleus and ventral putamen to a limited extent (Fig. 10, ar1, ar3) but arborize extensively in the lateral nucleus and lateral basal nucleus. After entering the amygdala, one branch courses caudorostrally and forms a terminal plexus within the lateral nucleus (Fig. 10, ar2). The other branch follows a relatively straight course that traverses the medial aspect of the lateral nucleus and the lateral basal nucleus, where it gives off more collaterals (Fig. 10, arrow at section 170) and leaves the majority of terminals, both in the terminal plexuses and en passant along the collaterals (Fig. 10, ar4-ar8). The latter branch also terminates to a limited extent in the accessory basal nucleus at a more rostral level but clearly spares the medial basal nucleus. In addition, axon 4-3 differs from axon 4-2 in that it does not have any collaterals projecting beyond the ventrocaudal striatum and the amygdala.

The main axonal trunks of two partially reconstructed TEav axons (one from case 3 and the other from case 4) also project to both the ventrocaudal striatum and the dorsolateral aspect of the deep amygdaloid nuclei. We also reconstructed one TEad axon that has five arbors in the ventrocaudal striatum and two arbors in the dorsolateral aspect of the deep amygdaloid nuclei (axon 2-1, Fig. 11).

\section{Dual projection of TEav axons to the amygdala and cortex}

As has been described in the previous section, labeled terminals in the medial basal nucleus of the amygdala were observed only after TEav injections. Figure 12 shows a TEav axon that projects to the medial basal nucleus (axon 4-4). On leaving the injection site, the main axonal trunk courses rostrally and medially and enters the white matter, where it gives off two initial branches (Fig. 12, arrow at section 145). One branch travels a short distance in the white matter, enters the cortex again, and forms two terminal arbors (Fig. 12, ar1, ar2) within the rostral portion of the perirhinal cortex (rostral division of area 36; Fig. 12, 36r) (for subdivisions of the perirhinal cortex, see Saleem and Tanaka, 1996). The terminals of both arbors are distributed from layer IV through layer I. The other branch continues its course medially and rostrally in the white matter lateral to the fundus of the rhinal sulcus and gives off two collaterals (Fig. 12, arrows at sections 204 and 219), which terminate again within 36r, with terminals extending across all the cortical layers (Fig. 12, ar3, ar4). It is of interest to note that except for one arbor, all arbors in 36r (Fig. 12, ar2-ar4) were reconstructed from the same rostrocaudally elongated dense terminal field (the "core region"; see Saleem and Tanaka, 1996). The main branch then turns upward, travels in the white matter around the fundus of the rhinal sulcus, and finally enters the medial basal nucleus of the amygdala, where it gives off two further collaterals that arborize completely within this nucleus (Fig. 12, ar5, ar6).

Figure 13 shows another TEav axon that has a collateral terminating in the medial basal nucleus (axon 4-5). This axon also bifurcates in the white matter (Fig. 13, arrow at section 184). The branch that projects to the medial basal nucleus follows course 


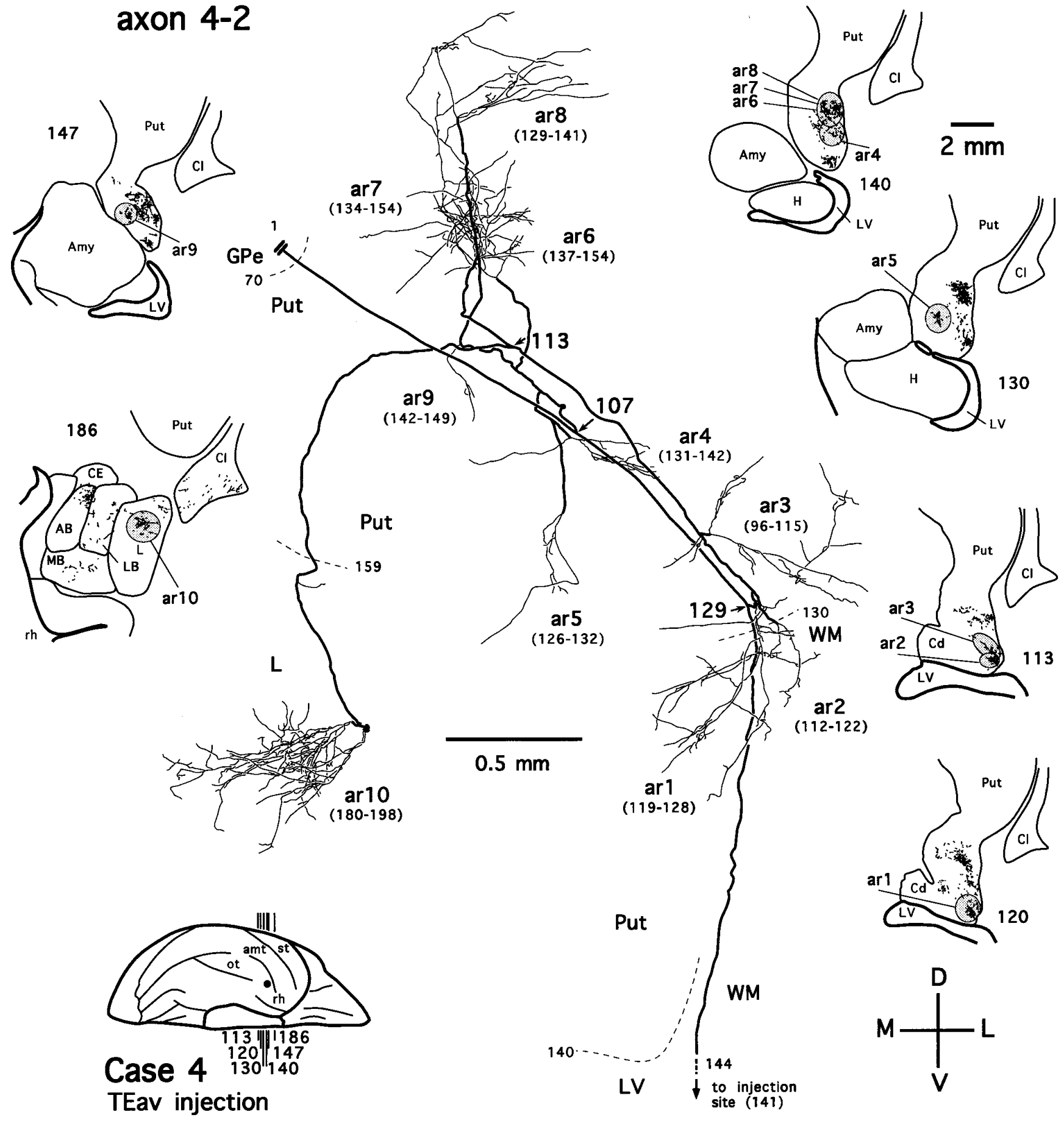

Figure 7. Camera-lucida reconstruction of axon 4-2 labeled by PHA-L anterogradely transported from TEav (case 4) to the ventrocaudal striatum and the dorsolateral aspect of the deep amygdaloid nuclei. Thick lines represent the main axonal trunk and collaterals, and thin lines represent terminal arbors. Numbers indicate serial numbers of individual sections; smaller numbers are posterior. This axon was serially reconstructed through 103 sections (section thickness, $40 \mu \mathrm{m}$ ). Ten terminal arbors (ar1-ar10) were drawn; each arbor is presented with a range of sections (numbers in parentheses), from which the arbor was completely reconstructed. Sections in which the axon gives off major collaterals are indicated by section numbers and arrows. Dashed lines (with section numbers) indicate borders between different structures. The global locations of terminal arbors are illustrated in low-magnification camera-lucida drawings of selected sections, where terminal clusters containing individual arbors are circumscribed by shaded ellipses or circles. Double lines indicate the incomplete portion of the axon. The main axonal trunk in the white matter was followed to the injection site. $A B$, Accessory basal nucleus; $C E$, central nucleus; $L$, lateral nucleus; $L B$, lateral basal nucleus; $M B$, medial basal nucleus; $A m y$, amygdala; $C d$, caudate nucleus; $C l$, claustrum; $G P e$, external globus pallidus; $H$, hippocampus; $L V$, lateral ventricle; $P$ ut, putamen; $W M$, white matter; $r h$, rhinal sulcus. Orientation: $M$, medial; $L$, lateral; $D$, dorsal; $V$, ventral. The same conventions and abbreviations are used for illustrating other reconstructed axons unless otherwise noted. 

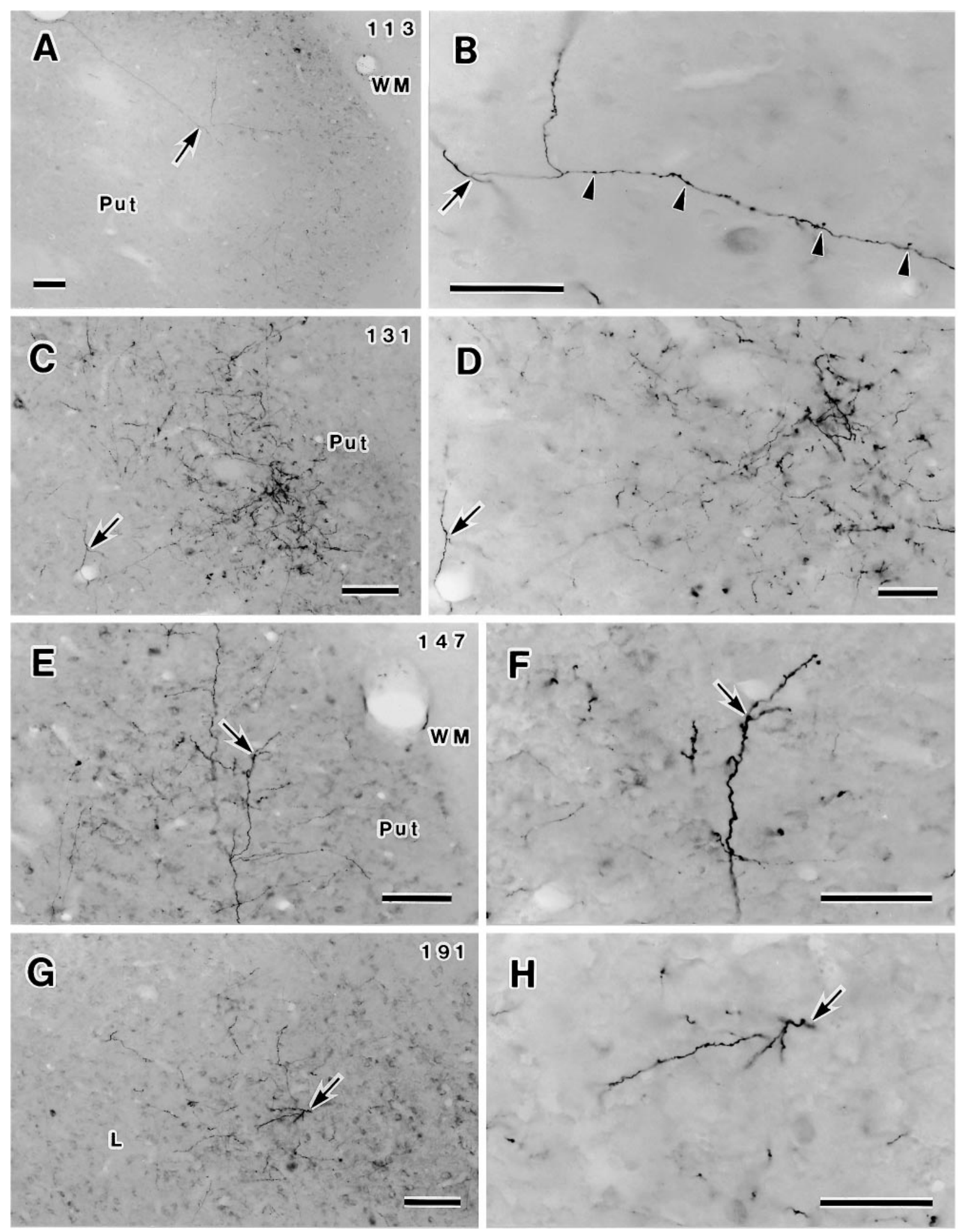

Figure 8. Photomicrographs showing axonal fibers and terminals labeled after PHA-L injection into TEav (case 4). Four sections (113, 131, 147, 191) are illustrated at both low (left column) and high (right column) magnifications. Arrows in each pair of photomicrographs indicate identical locations. A, $B$, Proximal axonal trunk and an emerging branch (arrow) of axon 4-2 in the ventral putamen (for complete details, see Figs. 7, ar3, 9B). Note the differences in thickness and morphology between the smooth proximal axonal and the beaded branch with terminal swellings and varicosities (arrowheads in $B$ ). $C, D$, Distinctive terminal cluster in the ventral putamen, in which two arbors of two different axons were reconstructed (axon 4-2, Figs. 7, $9 E$, ar8; axon 4-1, Fig. 15, ar6). The arrows indicate an emerging branch (of axon 4-1) coursing toward the densest part of the cluster. $E$, $F$, Arbor $a r 7$ of axon 4-2 (Figs. 7, 9D) in the ventral putamen. $G, H$, Circumscribed terminal cluster within the lateral nucleus $(L)$ of the amygdala. Arbor 10 of axon 4-2 (Figs. $7,9 F)$ was completely reconstructed in this cluster. $L$, Lateral nucleus of the amygdala; $P u t$, putamen; $W M$, white matter. Scale bars: $A, C, E, G, 100 \mu$ m; $B, D, F, H, 50 \mu \mathrm{m}$. 


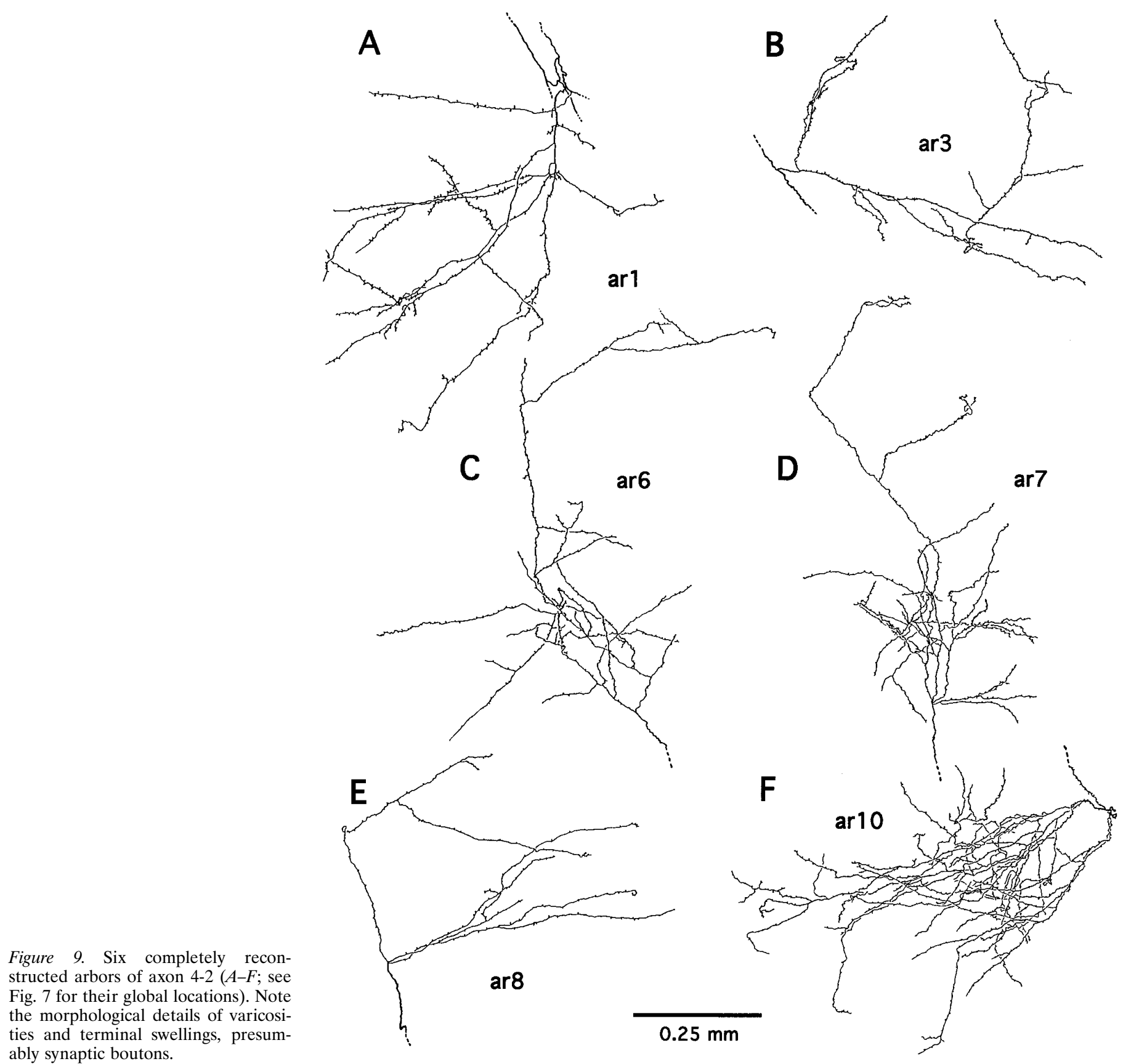
ably synaptic boutons.

similar to that of axon 4-4 and forms two terminal arbors (Fig. 13, ar1, ar2) that overlap with those given off by axon 4-4 in the medial basal nucleus (Figs. 12, 13, compare the camera-lucida drawings of sections 215 and 230). The other branch gives off two collaterals in the white matter (Fig. 13, arrow at section 245). One collateral courses rostrally and medially and breaks down into at least two terminal arbors that extend across all the cortical layers at different rostrocaudal levels of the perirhinal cortex (Fig. 13, ar5 in 36r, ar6 in the polar division of area 36, 36p) (see Saleem and Tanaka, 1996). The remaining finer branches of this collateral in the perirhinal cortex were not pursued further, because they run into the densely distributed terminal field, which was so dense that single axons could not be reliably reconstructed. The other collateral continues its course rostrally and ventrally and forms two arbors in the cytoarchitectonic area TG (Fig. 13, ar3, ar4). The terminals of both arbors are confined to the layers IV and III.
Four partially reconstructed TEav axons that we started to trace in the medial basal nucleus showed properties similar to those of the above two axons; they never terminate in the dorsolateral aspect of the deep amygdaloid nuclei, whereas they have collaterals that form arbors in the perirhinal cortex. Although we did not trace all the axons that project to the medial basal nucleus, the above evidence strongly suggests that (1) no axons project to both the dorsolateral aspect of the deep amygdaloid nuclei and the medial basal nucleus; and (2) all axons projecting from TEav to the medial basal nucleus also have collaterals projecting to the perirhinal cortex and/or area TG.

\section{Other corticostriatal and corticoamygdalar axons arising from} TEav and TEad

Of the remaining 13 completely reconstructed axons, seven project to the ventrocaudal striatum, four to the dorsolateral 


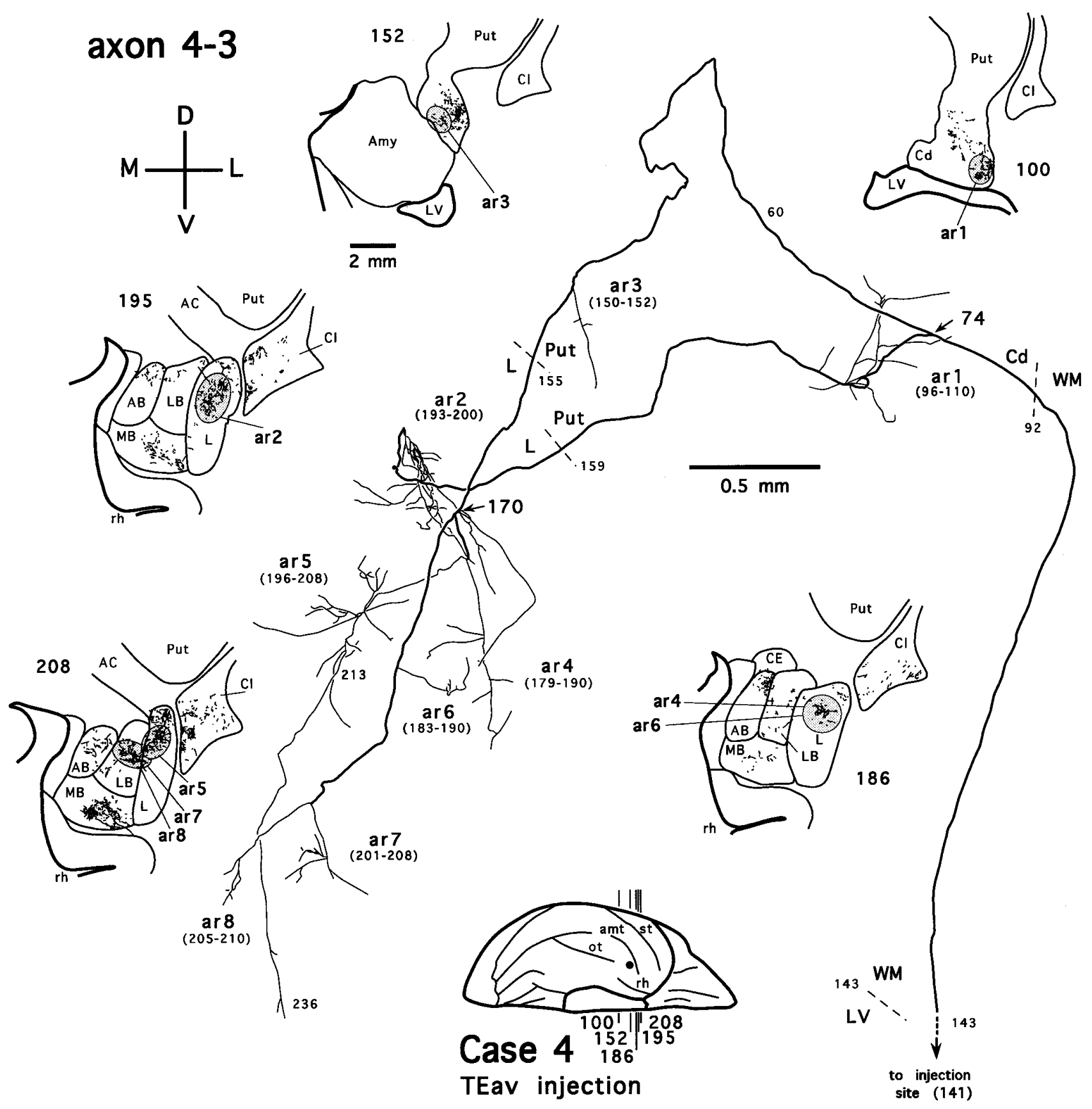

Figure 10. Camera-lucida reconstruction of axon 4-3 labeled by PHA-L anterogradely transported from TEav (case 4) to the ventrocaudal striatum and the dorsolateral aspect of the deep amygdaloid nuclei. This axon was serially reconstructed through 177 sections (section thickness, $40 \mu \mathrm{m}$ ). $A C$, Anterior commissure. Other abbreviations and conventions are the same as in Figure 7.

aspect of the deep amygdaloid nuclei, and the other two to the ventral striatum.

Figure 14 shows an axon reconstructed from a TEad case (axon 1-1). Several collaterals of this axon arborize in the tail of the caudate nucleus (Fig. 14, arl, ar2) and the ventral putamen (Fig. 14, ar3-ar6). The main axonal trunk continues its course dorsomedially and posteriorly, presumably toward the thalamic nuclei. This axon, without bearing any collaterals projecting to the amygdala, otherwise resembles axon 4-2 (Fig. 7) and may belong to those long-range axons that project beyond the thalamic level. Figure 15 illustrates a TEav axon (axon 4-1) reconstructed from the same case as axon 4-2 and axon 4-3. Like axon 4-2 and axon 4-3 (Figs. 7, 10), axon 4-1 also bif urcates after entering the ventral putamen and gives off collaterals that arborize within the ventral putamen (Fig. 15, ar1-ar6). Five other completely reconstructed axons, four from the TEav cases and one from one TEad case, also branch and arborize completely in the ventrocaudal striatum.

We also traced four axons that project exclusively to the dorsolateral aspect of the deep amygdaloid nuclei (two from the TEav cases and two from the TEad cases). Figure 16 shows one such axon, reconstructed from a TEav case (axon 3-7). The axon gives off two branches just before entering the amygdala (Fig. 16, 


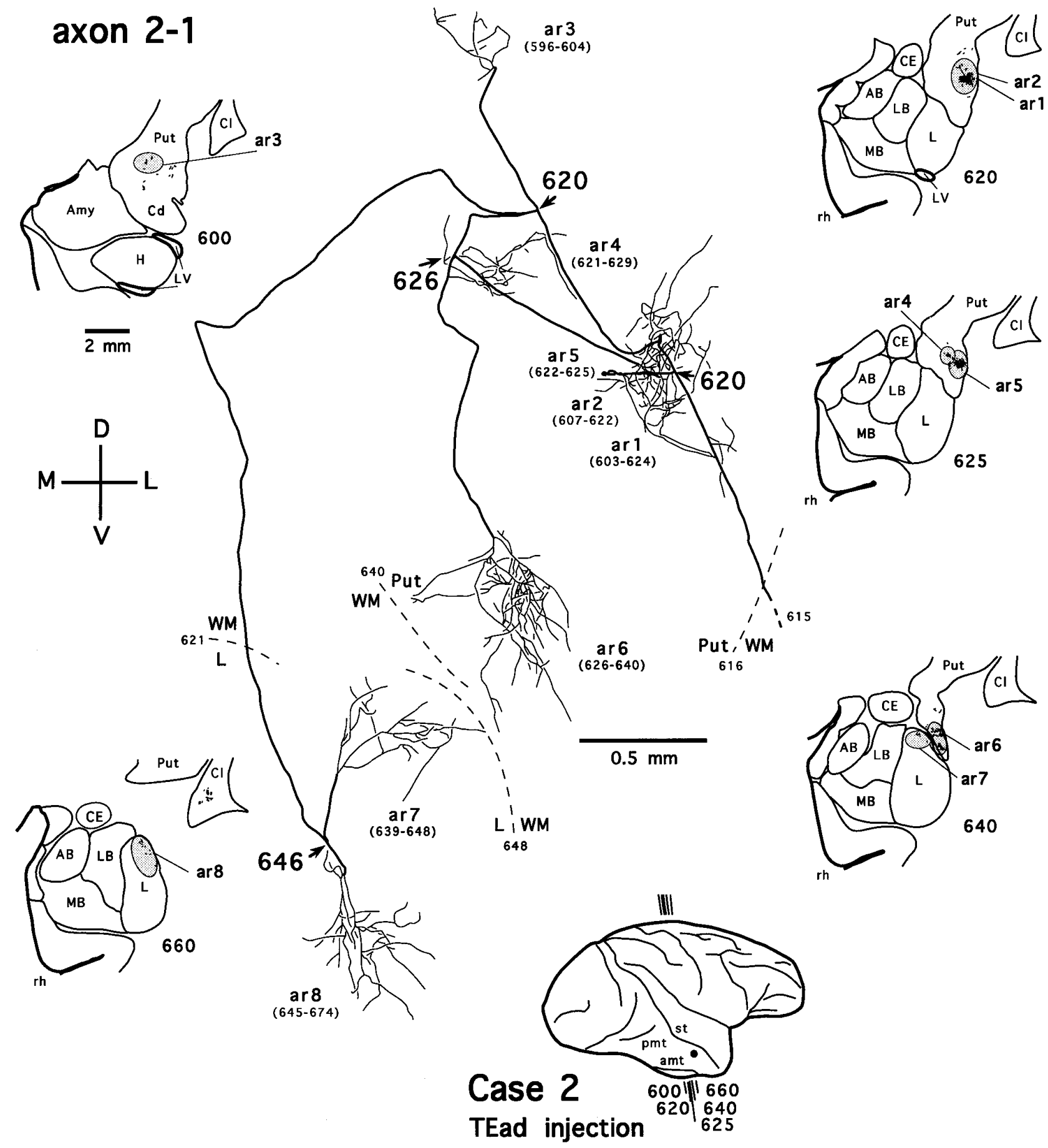

Figure 11. Camera-lucida reconstruction of axon 2-1 labeled by PHA-L anterogradely transported from TEad (case 2) to the ventrocaudal striatum and the dorsolateral aspect of the deep amygdaloid nuclei. This axon was serially reconstructed through 79 sections (section thickness, $35 \mu \mathrm{m}$ ). Abbreviations and conventions are the same as in Figure 7.

arrow at section 255). Both branches then run a long distance caudorostrally within the amygdala and give off seven arbors in the lateral nucleus and the lateral basal nucleus. Figure 17 shows a TEad axon (axon 2-2) that arborizes mostly in the lateral nucleus and the lateral basal nucleus of the amygdala but has an arbor in the basal nucleus of Meynert (Fig. 17, ar6). In general, the arborization patterns of single axons in the dorsolateral aspect of the deep amygdaloid nuclei were rather different be- tween TEad and TEav: TEad axons terminated within a limited spatial extent, whereas TEav axons ran along a relatively straight course caudorostrally. This heterogeneity of single axons in their spatial extents may underlie the difference in the caudorostral extents observed for the global distributions of terminals between TEad and TEav cases.

Two TEav axons in the ventral striatum were reconstructed. Figure 18 shows one such axon (axon 3-2). The main axonal trunk 


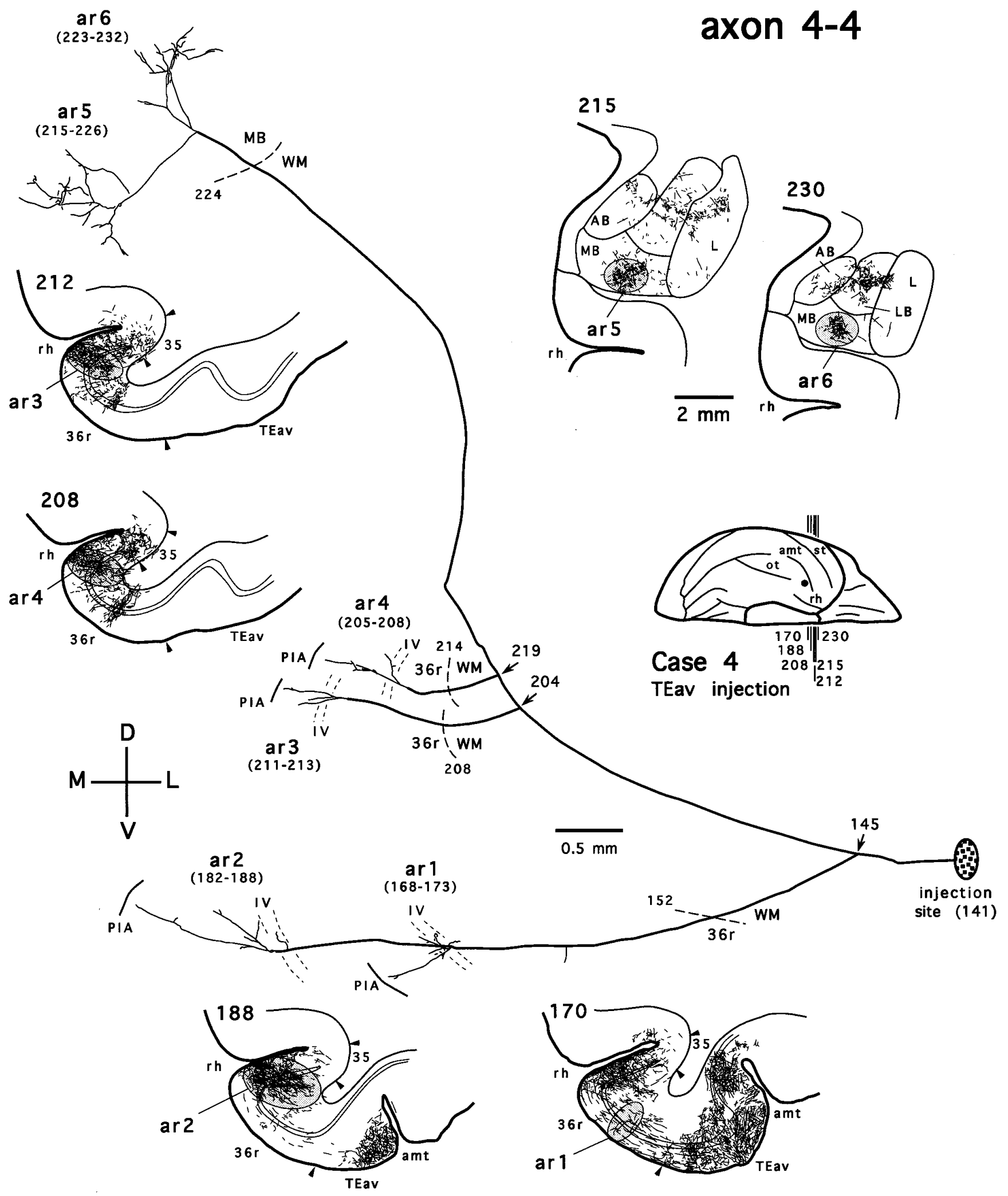

Figure 12. Camera-lucida reconstruction of axon 4-4 labeled by PHA-L anterogradely transported from TEav (case 4) to the medial basal nucleus (MB) of the amygdala and the perirhinal cortex (area 36). This axon was serially reconstructed through 92 sections (section thickness, $40 \mu \mathrm{m}$ ). Note that the branch terminating in the medial basal nucleus does not encroach into other deep amygdaloid nuclei. 35, Area 35; 36r, rostral division of area 36; amt, anterior middle temporal sulcus. Other abbreviations and conventions are the same as in Figure 7. 

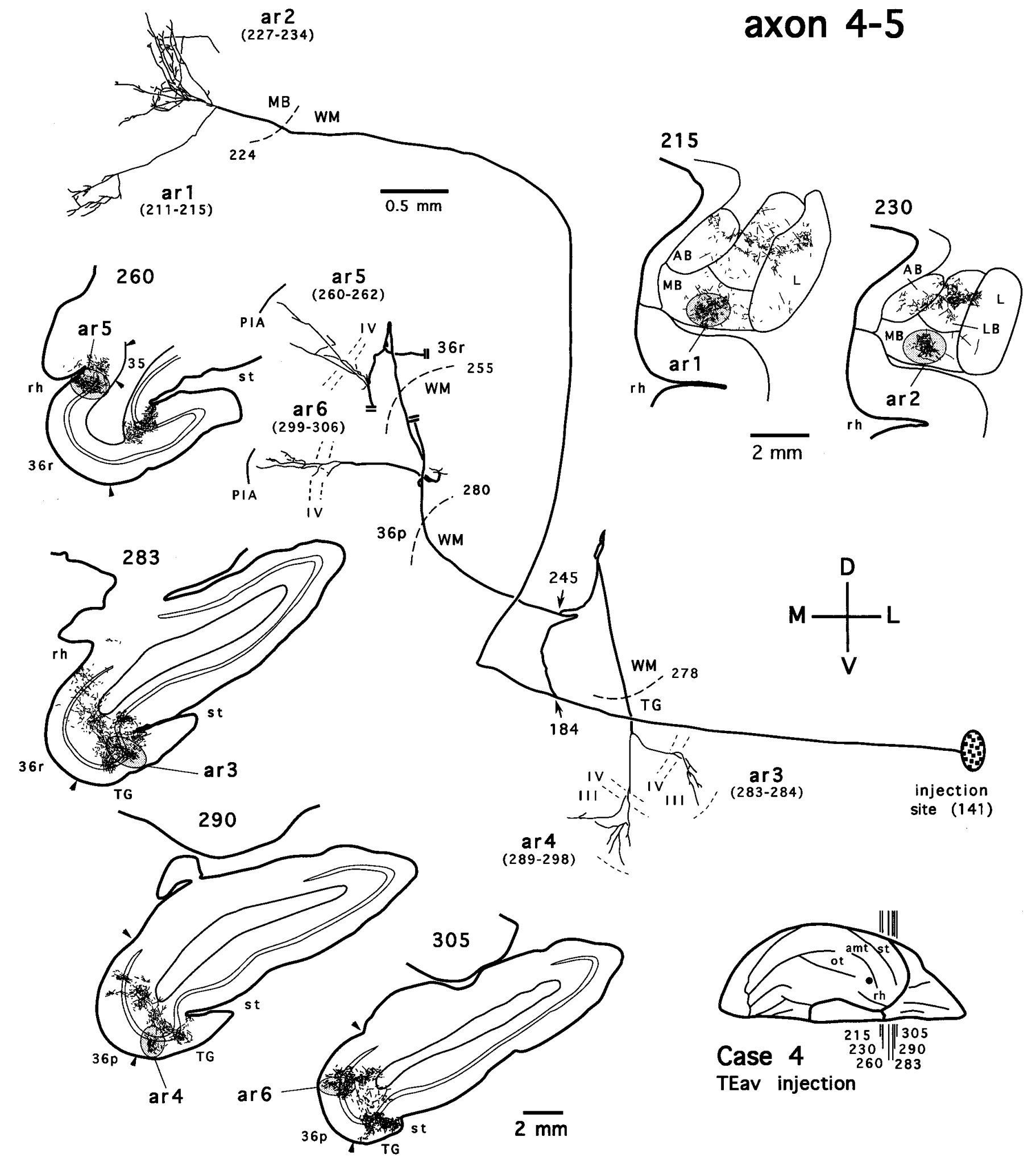

axon 4-5

Figure 13. Camera-lucida reconstruction of axon 4-5 labeled by PHA-L anterogradely transported from TEav (case 4) to the medial basal nucleus (MB) of the amygdala, the perirhinal cortex (area 36), and area TG. This axon was serially reconstructed through 166 sections (section thickness, $40 \mu \mathrm{m}$ ). Double lines show incomplete portions of the axon. 36p, Polar division of area 36; 36r, rostral division of area 36; TG, area TG; st, superior temporal sulcus. Other abbreviations and conventions are the same as in Figure 7. 


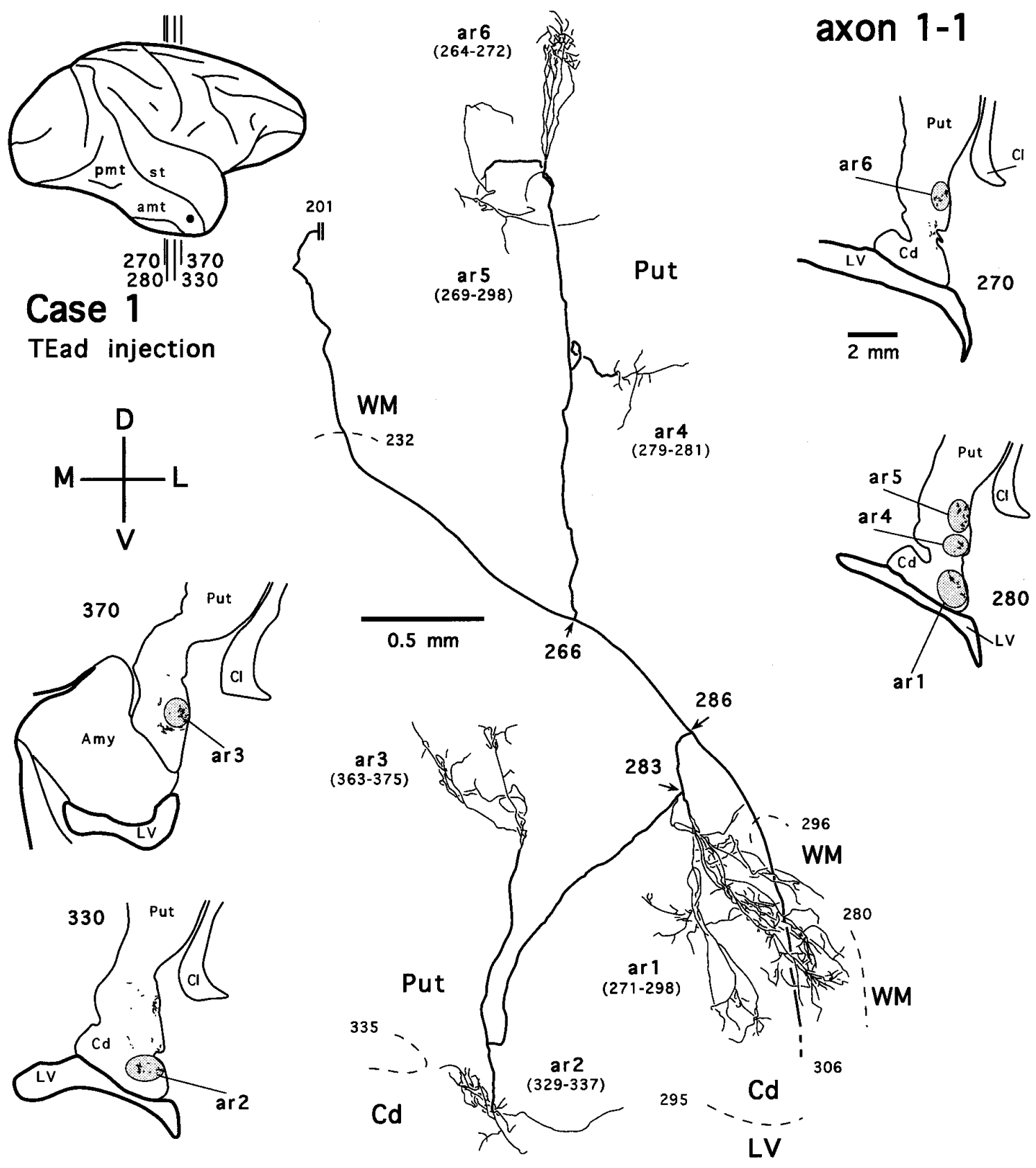

Figure 14. Camera-lucida reconstruction of axon 1-1 labeled by PHA-L anterogradely transported from TEad (case 1) to the ventrocaudal striatum. This axon was serially reconstructed through 112 sections (section thickness, $30 \mu \mathrm{m}$ ). Double lines indicate the incomplete portion of the axon. Abbreviations and conventions are the same as in Figure 7.

of this axon, along with that of the other simultaneously traced axon, was followed to the injection site. There was no indication that these axons bifurcate in the white matter, and their courses in the white matter are different from those projecting to the ventrocaudal striatum. The patchy distribution of terminal plexuses (Fig. 18, see camera-lucida drawings of sections 310, 330, 360) and multiple arbors (Fig. 18, ar1-ar7) of the axon, however, appear to be similar to those of the axons reconstructed in the ventrocaudal striatum.

\section{Corticostriatal projections: global terminal distribution} versus single axonal arbors

After a focal injection of PHA-L into either TEav or TEad, labeled terminals in the ventrocaudal striatum were distributed in a re- stricted territory that measured $\sim 1.8-2.6 \mathrm{~mm}$ mediolaterally, $3.5-$ $4.7 \mathrm{~mm}$ dorsoventrally, and $3.4-3.6 \mathrm{~mm}$ rostrocaudally. The labeled terminals in the territory are grouped into a small number of patches in any given coronal section (Figs. 2, 3; also see cameralucida drawings of coronal sections in Figs. 7, 10, 14, 15). By examining closely spaced sections it was revealed that the patches formed rostrocaudally elongated, rod-like modules (referred to as "rods" below). In one TEav case (case 4), as demonstrated in Figure $19 A$, there were six rods, each of which measured $0.8-2.8$ $\mathrm{mm}($ mean $\pm \mathrm{SD}, 1.63 \pm 0.66 \mathrm{~mm})$ in the rostrocaudal direction and $0.4-0.8 \mathrm{~mm}(0.65 \pm 0.15 \mathrm{~mm})$ in diameter in coronal sections. There were four rods in another TEav case (case 3) and five (case 1 ) and four (case 2) rods in the TEad cases. 


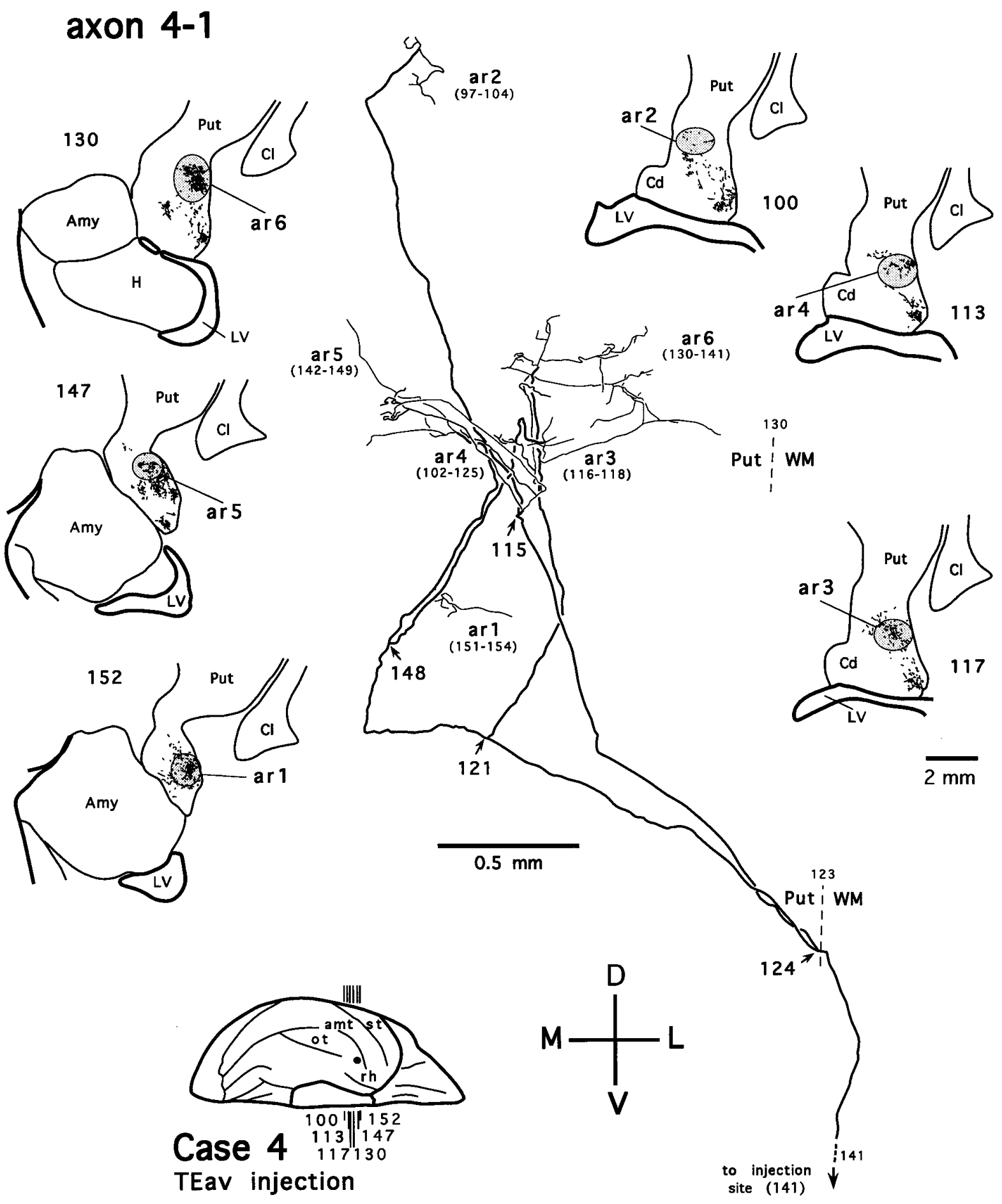

Figure 15. Camera-lucida reconstruction of axon 4-1 labeled by PHA-L anterogradely transported from TEav (case 4) to the ventrocaudal striatum. This axon was serially reconstructed through 58 sections (section thickness, $40 \mu \mathrm{m}$ ). Abbreviations and conventions are the same as in Figure 7.

The axons that projected to the ventrocaudal striatum were shown to bear multiple terminal arbors. Of the 10 reconstructed axons that arborized completely or had collaterals arborizing in the ventrocaudal striatum, the number of arbors within the ventrocaudal striatum ranged from two (axon 4-3, Fig. 10) to nine (axon 4-2, Fig. 7), with most of the axons having five to seven arbors (axon 1-1, Fig. 14; axon 4-1, Fig. 15). The complexity of ramifications among reconstructed arbors varied greatly (com- pare Figs. 7, 10, 14, 15). The well ramified arbors of both TEav and TEad axons (e.g., arbors of axon 4-2 shown in Fig. 9A-E) typically measured $0.4 \mathrm{~mm}$ in the minor axis and $0.8 \mathrm{~mm}$ in the major axis, whereas other arbors had a relatively simple appearance. There was no obvious tendency that individual arbors had any preferred orientations.

Single axons projected to multiple rod-like modules with variable degrees of divergence. Multiple arbors of an axon were 


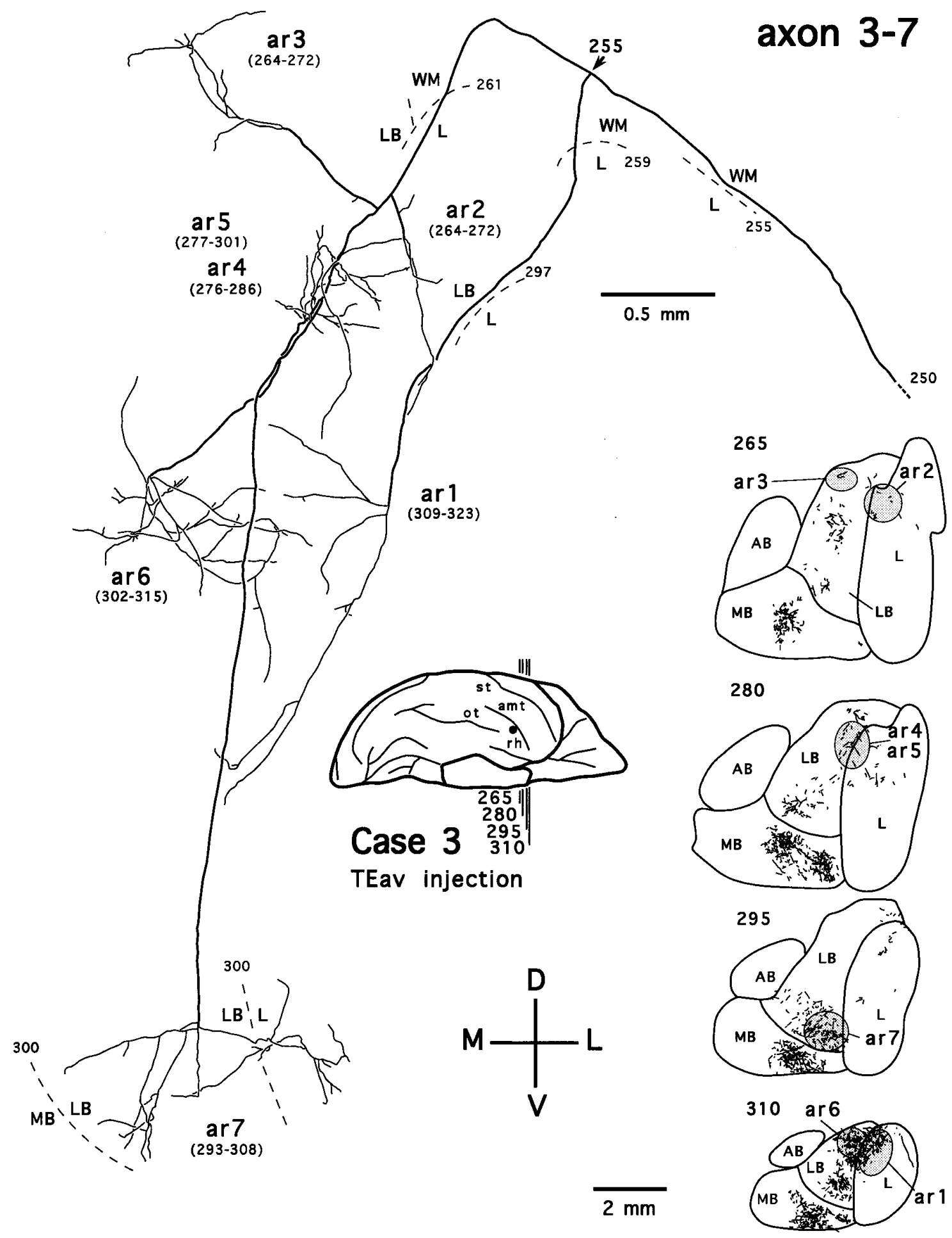

Figure 16. Camera-lucida reconstruction of axon 3-7 labeled by PHA-L anterogradely transported from TEav (case 3) to the dorsolateral aspect of the deep amygdaloid nuclei. This axon was serially reconstructed through 101 sections (section thickness, $40 \mu \mathrm{m}$ ). Note that although this axon innervates most of the dorsolateral aspect of the deep amygdaloid nuclei, it avoids encroaching into the medial basal nucleus (MB). Abbreviations and conventions are the same as in Figure 7.

usually segregated from each other and located in different rods, although in some cases more than one arbor of the same axon innervated a single rod at different rostrocaudal levels. For example, axon 1-1 innervated all five rods in case 1, and axon 2-1 innervated all four rods in case 2, whereas axons 4-1, 4-2, and 4-3 innervated three, two, and four of the six rods, respectively, in case 4 . Because of the limited number of rods, this divergent projection pattern of single axons also suggests that many single axons projecting from a small cortical site converge in a given rod. To demonstrate this convergence, arbors of the three axons 


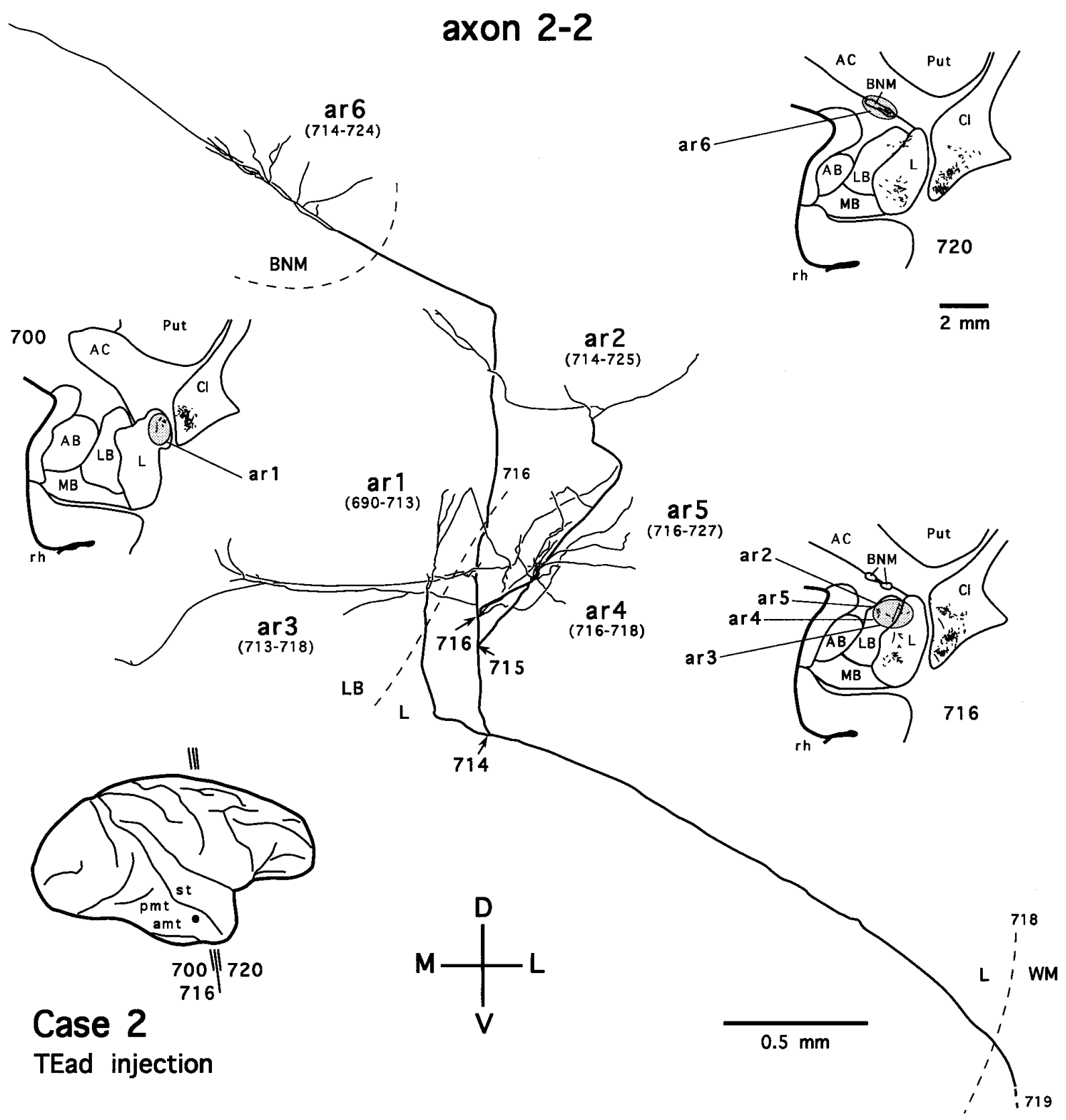

Figure 17. Camera-lucida reconstruction of axon 2-2 labeled by PHA-L anterogradely transported from TEad (case 2) to the dorsolateral aspect of the deep amygdaloid nuclei and the basal nucleus of Meynert. This axon was serially reconstructed through 38 sections (section thickness, $35 \mu \mathrm{m}$ ). BNM, Basal nucleus of Meynert. Other abbreviations and conventions are the same as in Figure 7.

reconstructed from the same TEav case (axons 4-1, 4-2, and 4-3) are illustrated in Figure $19 B$ to compare their distribution with the distribution of rods shown in Figure $19 A$. The rod marked by light pink is innervated by arbors of all the three axons, and the rod marked by blue is innervated by arbors of two axons (axons 4-1 and 4-2). The arbors of the three axons covered five of the six rods.

The terminal fields in the ventral striatum were also composed of rod-like modules, although they were less clearly separated from each other than those in the ventrocaudal striatum. However, it is obvious that single axons innervated multiple rods, as shown for one axon in Figure 18.

\section{DISCUSSION}

\section{Differential projections from TEad and TEav to the striatum and amygdala}

By focal injections of PHA-L, we have shown that TEad and TEav have different projections to the striatum and amygdala. Although TEad and TEav projected similarly to the ventrocaudal striatum, TEav projected additionally to the ventral striatum, to which TEad did not project. The projections from TEad and TEav to the dorsolateral aspect of the deep amygdaloid nuclei were different in their densities and topographic arrangements; the projections from TEav were more extensive than those from 

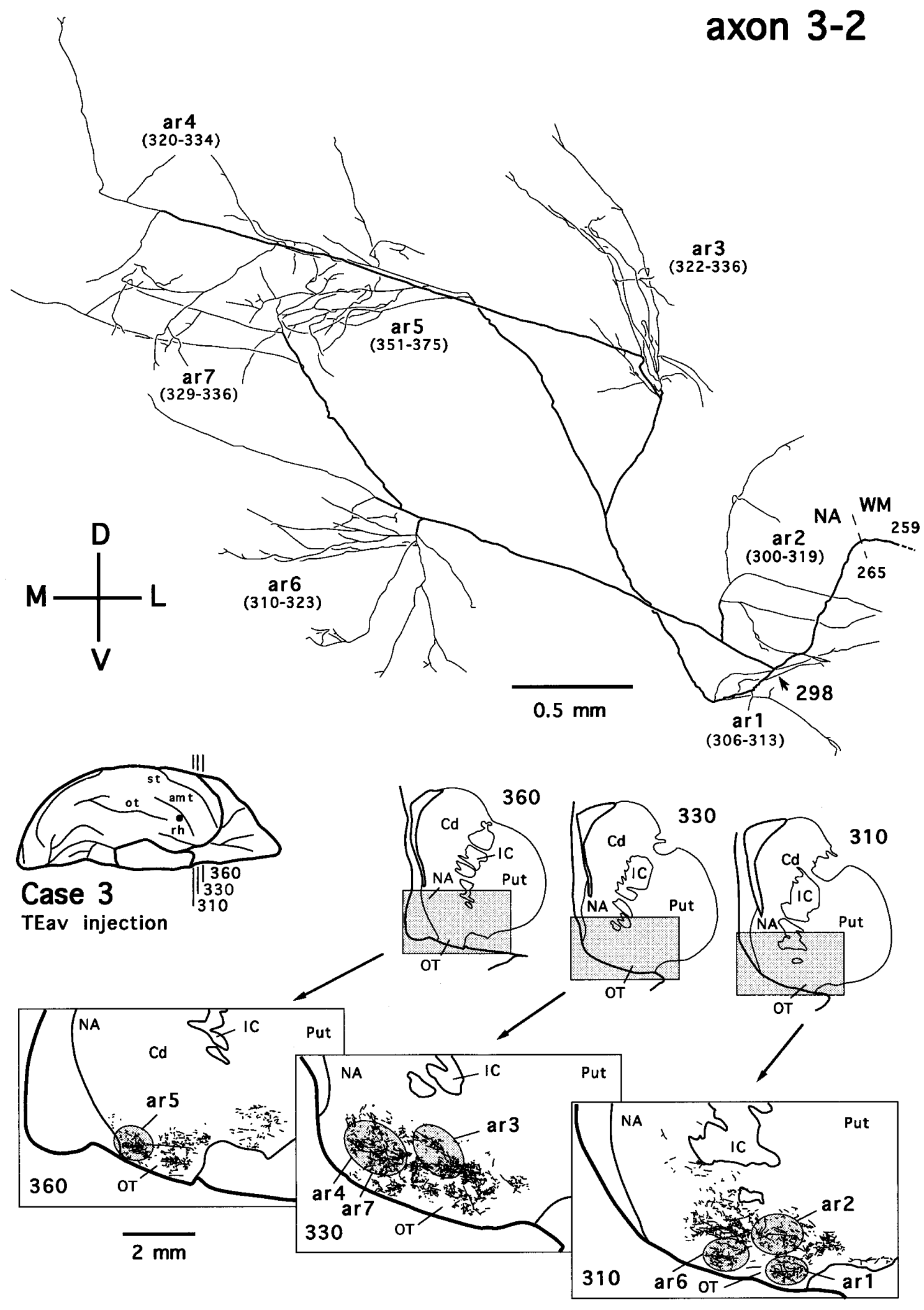

Figure 18. Camera-lucida reconstruction of axon 3-2 labeled by PHA-L anterogradely transported from TEav (case 3) to the ventral striatum. This axon was serially reconstructed through 197 sections (section thickness, $40 \mu \mathrm{m}$ ). $C d$, Caudate nucleus; $I C$, internal capsule; $N A$, nucleus accumbens; $O T$, olfactory tubercle; Put, putamen. Other abbreviations and conventions are the same as in Figure 7. 

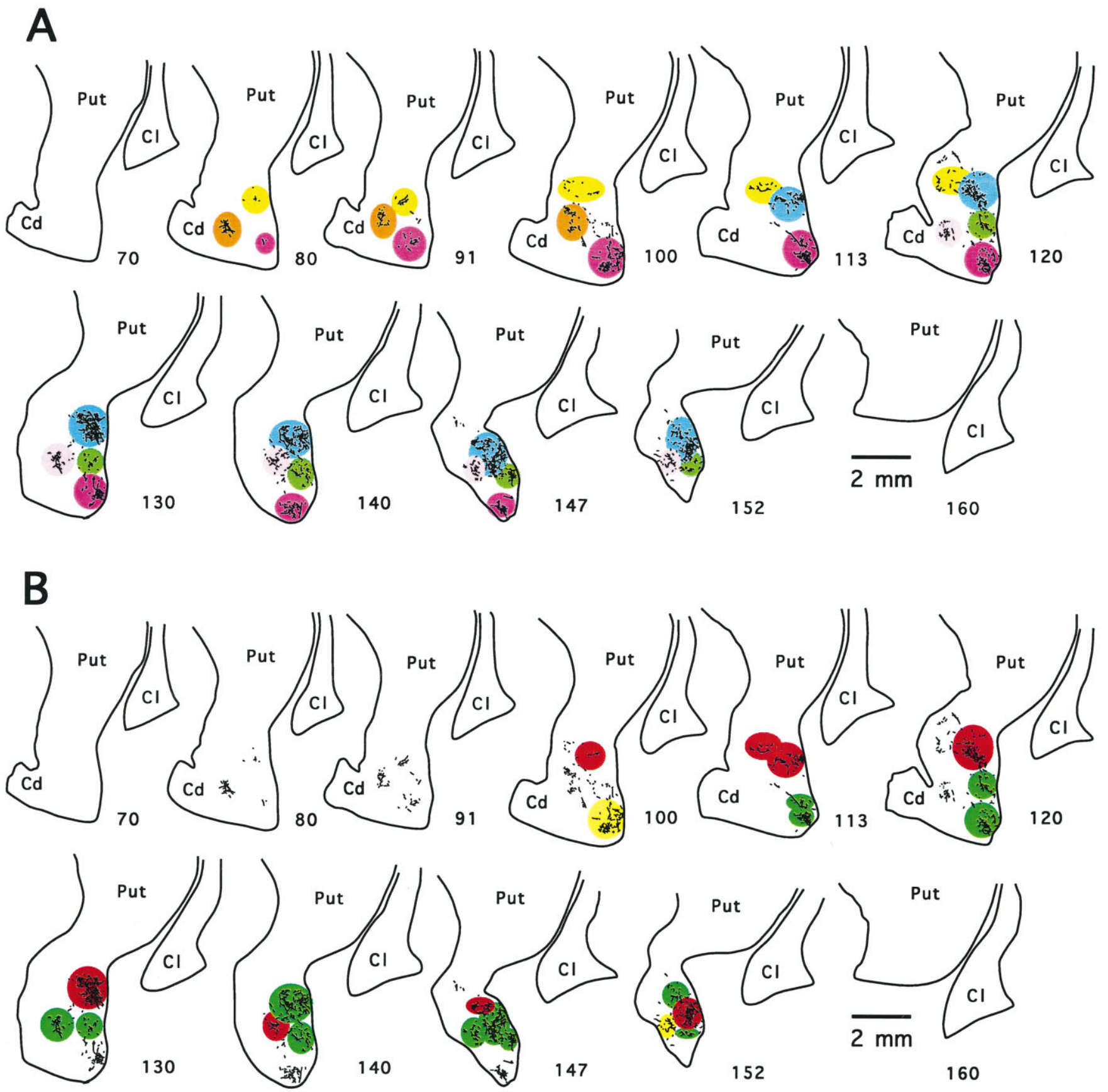

Case 4

TEav injection

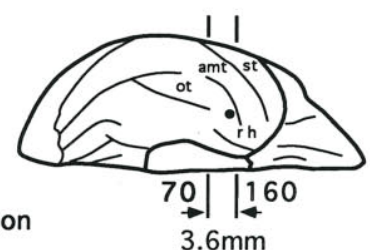

axon 4-1

axon 4-2

axon 4-3

Figure 19. Multiple rod-like modules of the terminal field and the distribution of terminal arbors of 3 completely reconstructed axons in the ventrocaudal striatum after PHA-L injection into TEav (case 4). Numbers indicate serial numbers of individual sections ( $40 \mu \mathrm{m}$ thick); bottom section numbers indicate posterior sections. $A$, Camera-lucida drawings of 11 coronal section outlines showing axonal terminals in the ventrocaudal striatum. Each rostrocaudally elongated rod-like module is illustrated in a different color. Six rods were observed in this case. $B$, Distribution of terminal arbors of 3 reconstructed axons [axon 4-1 (red), axon 4-2 (green), and axon 4-3 (yellow); see Figs. 7, 10, 15 for details of the 3 axons) in the terminal fields identical to those shown in $A$. Note that the arbors of the 3 axons innervated 5 of 6 rods, sparing only $1 \operatorname{rod}($ shown in brown in $A$ ). Cd, Caudate nucleus; $C l$, claustrum; Put, putamen. 
TEad, and the terminal field arising from TEav was distributed more medially than that from TEad. Finally, TEav projects to the medial basal nucleus, to which TEad did not project. These results, along with previous findings that TEad and TEav receive differential afferents from area TEO (Desimone et al., 1980; Martin-Elkins and Horel, 1992; Yukie et al., 1992) and send differential efferents to the prefrontal cortex (Saleem et al., 1995) and the perirhinal and entorhinal cortices (Saleem and Tanaka, 1996), indicate that the anterior part of TE is anatomically heterogeneous.

The present results regarding the projections from TEad and TEav to the ventrocaudal striatum and the dorsolateral aspect of the deep amygdaloid nuclei are generally consistent with previous results concerning the striatal and amygdalar projections from the middle temporal gyrus and inferior temporal gyrus (ITG) (Whitlock and Nauta, 1956; Jones and Powell, 1970; Kemp and Powell, 1970; Herzog and Van Hoesen, 1976; Van Hoesen et al., 1976, 1981; Yeterian and Van Hoesen, 1978; Aggleton et al., 1980; Turner et al., 1980; Iwai and Yukie, 1987; Iwai et al., 1987; Saint-Cyr et al., 1990; Webster et al., 1991, 1993; Baizer et al., 1993; for review, see Amaral et al., 1992; Parent and Hazrati, 1995). It has been noted that the ITG projects to the ventral striatum (Whitlock and Nauta, 1956; Van Hoesen et al., 1976, 1981; Yeterian and Van Hoesen; 1978) and the medial basal nucleus of the amygdala (Turner et al., 1980; Iwai and Yukie, 1987; Iwai et al., 1987). However, the large ablations or injections in the ITG in these previous studies appeared to have invaded the ectorhinal cortex, or area 36 (Brodmann, 1909), now considered the lateral portion of the perirhinal cortex (Amaral et al., 1987; Insausti et al., 1987; Suzuki and Amaral, 1994a,b; Saleem and Tanaka, 1996). Although the functional dissociation between TEav and the perirhinal cortex is still awaiting elucidation, recent results have shown that they are anatomically distinctive. TEav sends feed-forward projections to the perirhinal cortex (Suzuki and Amaral, 1994a,b; Saleem and Tanaka, 1996), and TEav and the perirhinal cortex project differentially to several areas in the medial temporal lobe, including the entorhinal cortex (Van Hoesen and Pandya, 1975; Suzuki and Amaral, 1994a,b; Saleem and Tanaka, 1996) and the hippocampus (Suzuki and Amaral, 1990). The present study contributes to our understanding of the projections from the anatomically defined TEav to the striatum and amygdala. Additional studies are needed to investigate the differences between the projections to these two structures from TEav and the perirhinal cortex, respectively.

\section{Parallel processing of visual cortical information in the striatum and amygdala via axon collaterals}

The present study represents the first attempt to elucidate corticostriatal and corticoamygdalar projections in monkeys by reconstructing single axons. We found that some single axons project to two or more subcortical structures or cortical areas. These include the axons projecting to the ventrocaudal striatum and the dorsolateral aspect of the deep amygdaloid nuclei and the axons projecting to the medial basal nucleus of the amygdala and area 36 of the perirhinal cortex or area TG. These axons may play functionally important roles in synchronizing the activities of multiple subcortical or cortical structures to which they feed common information from the visual cortex.

\section{Functional dissociation of the deep amygdaloid nuclei}

One of the most interesting findings in the present study is that fibers projecting to the medial basal nucleus are collaterals of the axons projecting to the perirhinal cortex or area TG, and these axons are separated from those projecting to the remaining deep amygdaloid nuclei. A definite number of the latter axons also have collaterals projecting to the ventrocaudal striatum. These findings raise the possibility that the dorsolateral aspect of the deep amygdaloid nuclei, including the lateral nucleus, lateral basal nucleus, and accessory basal nucleus, is functionally more closely related to the ventrocaudal striatum, whereas the medial basal nucleus is more closely related to the perirhinal cortex.

Other anatomical connections of the deep amygdaloid nuclei support this view. The lateral basal nucleus of the amygdala projects strongly to the ventrocaudal striatum (Parent et al., 1983; Russchen et al., 1985). Although most of the deep amygdaloid nuclei have reciprocal connections with the perirhinal cortex, the projections to the perirhinal cortex appear to be the strongest from the medial basal nucleus (Amaral and Price, 1984; Stefanacci et al., 1996). In addition, the medial basal nucleus receives projections from the entorhinal cortex (Amaral et al., 1992) and has reciprocal connections with the subiculum and the CA1 region of the hippocampus (Rosene and Van Hoesen, 1977; Amaral and Cowan, 1980; Amaral, 1986; Saunders et al., 1988).

Behavioral studies on lesioned monkeys have indicated different functional roles of the perirhinal cortex and the ventrocaudal striatum. The perirhinal cortex plays important roles in visual recognition memory (Meunier et al., 1993; Eacott et al., 1994; Gaffan, 1994; Ramus et al., 1994; Leonard et al., 1995; Tang and Aigner, 1996) and certain types of stimulus-stimulus association memories (Murray, 1996). The ventrocaudal striatum has been suggested to be involved in stimulus-response association learning or habit formation (Mishkin et al., 1984; Petri and Mishkin, 1984). A more recent view suggests that this pathway is generally important for the control of action (Gaffan, 1996). Thus, although the amygdala is known to be crucial in relating environmental stimuli to primary reinforcement (Gaffan, 1992) and emotional memory (LeDoux, 1992; Gallagher and Chiba, 1996), it is possible that the dorsolateral aspect of the deep amygdaloid nuclei is more related to stimulus-response association or habit formation, whereas the medial basal nucleus is more related to stimulusstimulus association or recognition memory.

\section{Organization of the corticostriatal projection}

The detailed organization of the corticostriatal projection is of the utmost importance in determining the nature of the information that is conveyed and integrated through the cortico-basal ganglia-thalamo-cortical loop (Alexander et al., 1986; Parent and Hazrati, 1995). The patchy distributions of terminal fields have been observed for projections from other cortical areas to the striatum (Künzle, 1975; Goldman and Nauta, 1977; Jones et al., 1977; Selemon and Goldman-Rakic, 1985; Eblen and Graybiel, 1995), and they are related to the patch matrix striatal compartmentalizations (Graybiel, 1990; Gerfen, 1992; Parent and Hazrati, 1995). However, a consensus on the plausible functional roles of these divergent corticostriatal projections has not been established. Some investigators link them to the "parallel processing" in the cortico-basal ganglia-thalamocortical loop (Alexander et al., 1986; Alexander and Crutcher, 1990), whereas others favor an "informational funnel hypothesis" (Percheron and Filion, 1991; but see Parent and Hazrati, 1993). To resolve the differences in the interpretation of functional roles of divergent corticostriatal projections, studies at the cellular level are needed. The few previous studies on the corticostriatal projections at the singlecell level have substantially underestimated the complexity of 
divergent arborizations of single axons (Ramón y Cajal, 1911; DiFiglia et al., 1978), which led to the hypothesis that corticostriatal axons are poorly branched and emit boutons en passant to make synaptic contacts with striatal neurons along the rostrocaudal plane of the striatum (see Parent and Hazrati, 1995, their Fig. 2C).

Based on single-unit and optical recording experiments (Fujita et al., 1992; Wang et al., 1996), it is known that cells located in a column (equivalent to the size of our injections) in the anterior TE tend to respond to related but not identical visual features of objects. It is proposed that TE cells organized in this way may underlie the flexible coding of visual features of objects, i.e., the toleration of tiny changes in the visual patterns caused by changes in viewing angle, viewing distance, or luminance (Tanaka, 1993, 1996). This invariance, however, requires that single efferent axons from a TE column, which represent slightly different visual features, converge to single cells in the downstream. We showed that the projections from TE to the ventrocaudal striatum had characteristics consistent with this requirement. Although the projection from a TE column diverges to several rod-like modules in the ventrocaudal striatum, each of the rod-like modules is innervated by a large number of axons projecting from the TE column. It is likely that the variations in information represented in a TE column are integrated in each module. We would further suggest that these modules may form a striatal functional unit. Assuming that these modules, to which a TE column divergently projects, receive different kinds of information from other sensory cortical areas and the amygdala individually, whereas their outputs converge onto a single site in the pallidum or the substantia nigra, the functional unit will work as machinery for associating visual information represented in a cortical column with massive amounts of information from other sources. This may be a general rule for the organization of corticostriatal projections. Indeed, in a study performed by placing anterograde tracers in the sensorimotor cortex and retrograde tracers in the external and internal pallidal segments, Flaherty and Graybiel (1994) have shown that dispersed modules ("matriosomes") in the putamen, which receive projections from a single site in the sensorimotor cortex, in turn send convergent outputs to a single site in the pallidum.

\section{REFERENCES}

Aggleton JP, Burton MJ, Passingham RE (1980) Cortical and subcortical afferents to the amygdala of the rhesus monkey (Macaca mulatta). Brain Res 190:347-368.

Alexander GE, Crutcher MD (1990) Functional architecture of basal ganglia circuit: neural substrates of parallel processing. Trends Neurosci 13:266-271.

Alexander GE, DeLong MR, Strick PL (1986) Parallel organization of functionally segregated circuits linking basal ganglia and cortex. Annu Rev Neurosci 9:357-381.

Amaral DG (1986) Amygdalohippocampal and amygdalocortical projections in the primate brain. In: Excitatory amino acids and epilepsy (Schwarcz R, Ben-Ari Y, eds), pp 3-17. New York: Plenum.

Amaral DG, Cowan WM (1980) Subcortical afferents to the hippocampal formation in the monkey. J Comp Neurol 189:573-591.

Amaral DG, Price JL (1984) Amygdalo-cortical projections in the monkey (Macaca fascicularis). J Comp Neurol 230:465-496.

Amaral DG, Insausti R, Cowan WM (1987) The entorhinal cortex of the monkey. I. Cytoarchitectonic organization. J Comp Neurol 264:326-355.

Amaral DG, Price JL, Pitkanen A, Carmichael ST (1992) Anatomical organization of the primate amygdaloid complex. In: The amygdala: neurobiological aspects of emotion, memory, and mental dysfunction (Aggleton JP, ed), pp 1-66. New York: Wiley.

Baizer JS, Desimone R, Ungerleider LG (1993) Comparison of subcor- tical connections of inferior temporal and posterior parietal cortex in monkey. Vis Neurosci 10:59-72.

Brodmann K (1909) Vergleichende Lokalisationslehre der Grosshirnrinde: in ihren Prinzipien dargestellt anf Grund des Zellenbaues. Leipzig: Verlag von Johann Ambrosius Barth.

Cheng K, Saleem KS, Tanaka K (1993) PHA-L study of the subcortical projections of the macaque inferotemporal cortex. Soc Neurosci Abstr 19:971.

Dean P (1976) Effects of inferotemporal lesions on the behavior of monkeys. Psychol Bull 83:41-71.

Desimone R, Fleming J, Gross CG (1980) Prestriate afferents to inferior temporal cortex: an HRP study. Brain Res 184:41-55.

DiFiglia M, Pasik T, Pasik P (1978) A Golgi study of afferent fibers in the neostriatum of monkeys. Brain Res 152:341-347.

Eacott MJ, Gaffan D, Murray EA (1994) Preserved recognition memory for small sets, and impaired stimulus identification for large sets, following rhinal cortex ablations in monkeys. Eur $\mathrm{J}$ Neurosci 6:1466-1478.

Eblen F, Graybiel AM (1995) Highly restricted origin of prefrontal cortical inputs to striosomes in the macaque monkeys. J Neurosci 15: 5999-6013.

Flaherty AW, Graybiel AM (1994) Input-output organization of the sensorimotor striatum in the squirrel monkey. J Neurosci 14:599-610.

Fujita I, Tanaka K, Ito M, Cheng K (1992) Columns of visual features of objects in monkey inferotemporal cortex. Nature 360:343-346.

Gaffan D (1992) Amygdala and the memory of reward. In: The amygdala: neurobiological aspects of emotion, memory, and mental dysfunction (Aggleton JP, ed), pp 471-483. New York: Wiley.

Gaffan D (1994) Dissociated effects of perirhinal cortex ablation, fornix transection and amygdalectomy: evidence for multiple memory systems in the primate temporal pole. Exp Brain Res 99:411-422.

Gaffan D (1996) Memory, action and the corpus striatum: current developments in the memory-habit distinction. Semin Neurosci 8:33-38.

Gallagher M, Chiba AA (1996) The amygdala and emotion. Curr Opin Neurobiol 6:221-227.

Gerfen CR (1992) The neostriatal mosaic: multiple levels of compartmental organization. Trends Neurosci 15:133-139.

Gerfen CR, Sawchenko PE (1984) An anterograde neuroanatomical tracing method that shows the detailed morphology of neurons, their axons and terminals: immonuhistochemical localization of an axonally transported plant Phaseolous vulgaris leucoagglutinin (PHA-L). Brain Res 290:219-238.

Goldman PS, Nauta WJH (1977) An intricately patterned prefrontocaudate projection in the rhesus monkey. J Comp Neurol 171:369-386.

Graybiel AM (1990) Neurotransmitters and neuromodulators in the basal ganglia. Trends Neurosci 13:244-254.

Gross CG (1973) Visual functions of inferotemporal cortex. In: Handbook of sensory physiology, Vol VIII, Part 3B (Jung R, ed), pp 451482. Berlin: Springer.

Herzog AG, Van Hoesen GW (1976) Temporal neocortical afferent connections to the amygdala in the rhesus monkey. Brain Res 115:57-69.

Horel JA (1996) Perception, learning and identification studies with reversible suppression of cortical visual areas in monkeys. Behav Brain Res 76:199-214.

Insausti R, Amaral DG, Cowan WM (1987) The entorhinal cortex of the monkey. II. Cortical afferents. J Comp Neurol 264:356-395.

Iwai E, Yukie M (1987) Amygdalofugal and amygdalopetal connections with modality-specific visual cortical areas in macaques (Macaca fuscata, M. mulatta, and M. fascicularis). J Comp Neurol 261:362-387.

Iwai E, Yukie M, Suyama H, Shirakawa S (1987) Amygdalar connections with middle and inferior temporal gyri of the monkey. Neurosci Lett 83:25-29.

Jones EG, Powell TPS (1970) An anatomical study of converging sensory pathways within the cerebral cortex of monkey. Brain Res 93:793-378.

Jones EG, Coulter JD, Burton H, Porter R (1977) Cells of origin and terminal distribution of corticostriatal fibers arising in the sensorymotor cortex of monkeys. J Comp Neurol 173:53-80.

Kemp JM, Powell TPS (1970) The cortico-striate projection in the monkey. Brain 93:535-546.

Künzle H (1975) Bilateral projections from precentral motor cortex to the putamen and other parts of the basal ganglia. An autoradiographic study in Macaca fascicularis. Brain Res 88:195-209.

LeDoux JE (1992) Emotion and the amygdala. In: The amygdala: neu- 
robiological aspects of emotion, memory, and mental dysfunction (Aggleton JP, ed), pp 339-351. New York: Wiley.

Leonard BW, Amaral DG, Squire LR, Zola-Morgan S (1995) Transient memory impairment in monkeys with bilateral lesions of the entorhinal cortex. J Neurosci 15:5637-5659.

Martin-Elkins CL, Horel JA (1992) Cortical afferents to behaviorally defined regions of the inferior temporal and parahippocampal gyri as demonstrated by WGA-HRP. J Comp Neurol 321:177-192.

Meunier M, Bachevalier J, Mishkin M, Murray EA (1993) Effects on visual recognition of combined and separate ablations of the entorhinal and perirhinal cortex in the rhesus monkeys. J Neurosci 13:5418-5432.

Mishkin M, Malamut B, Bachevalier J (1984) Memories and habits: two neural systems. In: Neurobiology of learning and memory (Lynch $\mathrm{G}$, McGaugh JL, Weinberger NM, eds), pp 65-67. New York: Guilford.

Murray EA (1996) What have ablation studies told us about the neural substrates of stimulus memory? Semin Neurosci 8:13-22.

Parent A, Hazrati L-N (1993) Anatomical aspects of information processing in primate basal ganglia. Trends Neurosci 16:111-116.

Parent A, Hazrati L-N (1995) Functional anatomy of the basal ganglia. I. The cortico-basal ganglia-thalamo-cortical loop. Brain Res Rev 20:91-127.

Parent A, Mackey A, DeBellefeuille L (1983) The subcortical afferents to caudate nucleus and putamen in primate: a fluorescence retrograde double labeling study. Neuroscience 10:1137-1150.

Percheron G, Filion M (1991) Parallel processing in the basal ganglia: up to a point [letter]. Trends Neurosci 14:55-56.

Petri HL, Mishkin M (1984) Behaviorism, cognitivism, and the neuropsychology of memory. Am Sci 82:30-37.

Ramón y Cajal S (1911) Histologie du systeme nerveux de l'homme et des vertébrés, 2 Vol (translated by Azoulay L). Paris: Maloine.

Ramus SJ, Zola-Morgan S, Squire LR (1994) Effects of lesions of perirhinal cortex or parahippocampal cortex on memory in monkey. Soc Neurosci Abstr 20:1074.

Rockland KS, Saleem KS, Tanaka K (1994) Divergent feedback connections from areas V4 and TEO in the macaque. Vis Neurosci 11:579-600.

Rosene DL, Van Hoesen GW (1977) Hippocampal efferents reach widespread regions of the cerebral cortex and amygdala in the rhesus monkey. Science 198:315-317.

Russchen DT, Bakst I, Amaral DG, Price J (1985) The amygdalostriatal projections in the monkey. An anterograde tracing study. Brain Res 329:241-257.

Saint-Cyr JA, Ungerleider LG, Desimone R (1990) Organization of visual cortical inputs to the striatum and subsequent outputs to the pallido-nigral complex in the monkey. J Comp Neurol 298:129-156.

Saleem KS, Tanaka K (1996) Divergent projections from the anterior inferotemporal area TE to the perirhinal and entorhinal cortices in the macaque monkey. J Neurosci 16:4757-4775.

Saleem KS, Tanaka K, Rockland KS (1993) Specific and columnar projection from area TEO to TE in the macaque inferotemporal cortex. Cereb Cortex 3:454-464.

Saleem KS, Cheng K, Suzuki W, Tanaka K (1995) Differential cortical projection of dorsal and ventral sub-regions of the area TE in the macaque inferotemporal cortex. Int Brain Res Org Abstr 4:284.

Saunders RC, Rosene DL, Van Hoesen GW (1988) Comparison of the efferents of the amygdala and the hippocampal formation in the rhesus monkey. II. Reciprocal and non-reciprocal connections. J Comp Neurol 271:185-207.

Selemon LD, Goldman-Rakic PS (1985) Longitudinal topography and interdigitation of corticostriatal projections in the rhesus monkey. J Neurosci 5:776-794.

Stefanacci L, Suzuki WA, Amaral DG (1996) Organization of connections between the amygdaloid complex and the perirhinal and parahippocampal cortices in macaque monkeys. J Comp Neurol 375:552-582.

Suzuki WA, Amaral DG (1990) Cortical inputs to the CA1 field of the monkey hippocampus originate from the perirhinal and parahippocampal cortex but not from area TE. Neurosci Lett 115:43-48.

Suzuki WA, Amaral DG (1994a) Topographic organization of the reciprocal connections between the monkey entorhinal cortex and the perirhinal and parahippocampal cortices. J Neurosci 14:1856-1877.

Suzuki WA, Amaral DG (1994b) Perirhinal and parahippocampal cortices of the macaque monkey: cortical afferents. J Comp Neurol 350:497-533.

Tanaka K (1993) Neuronal mechanisms of object recognition. Science 262:685-688.

Tanaka K (1996) Inferotemporal cortex and object vision. Annu Rev Neurosci 19:109-139.

Tang Y, Aigner TG (1996) Muscarinic receptor blockade in perirhinal cortex impairs visual recognition memory in monkeys. Soc Neurosci Abstr 22:1119.

Turner BH, Mishkin M, Knapp M (1980) Organization of the amygdalopetal projections from modality-specific cortical association areas. J Comp Neurol 191:515-543.

Van Hoesen GW, Pandya DN (1975) Some connections of the entorhinal (area 28) and perirhinal (area 35) cortices of the rhesus monkey. I. Temporal lobe afferents. Brain Res 95:1-24.

Van Hoesen GW, Mesulam M-M, Haaxma R (1976) Temporal cortical projections to the olfactory tubercle in the rhesus monkey. Brain Res 109:375-381.

Van Hoesen GW, Yeterian EH, Lavizzo-Mourney R (1981) Widespread corticostriate projections from temporal cortex of the rhesus monkey. J Comp Neurol 199:205-219.

Wang G, Tanaka K, Tanifuji M (1996) Optical imaging of functional organization in the monkey inferotemporal cortex. Science 272:1665-1668.

Webster MJ, Ungerleider LG, Bachevalier J (1991) Connections of inferior temporal areas TE and TEO with medial temporal-lobe structures in infant and adult monkeys. J Neurosci 11:1095-1116.

Webster MJ, Bachevalier J, Ungerleider LG (1993) Subcortical connections of inferior temporal areas TE and TEO in macaque monkeys. J Comp Neurol 335:73-91.

Whitlock DG, Nauta WJH (1956) Subcortical projections from the temporal neocortex in Macaca mulatta. J Comp Neurol 106:183-212.

Yeterian EH, Van Hoesen GW (1978) Cortico-striate projections in the Rhesus monkey: the organization of certain cortico-caudate connections. Brain Res 139:43-63.

Yukie M, Hikosaka K, Iwai E (1992) organization of cortical visual projections to the dorsal and ventral parts of area TE of the inferotemporal cortex in macaques. Soc Neurosci Abstr 18:294. 